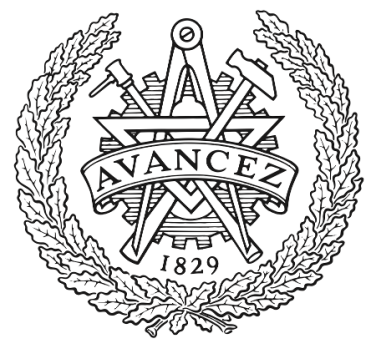

CHALMERS

UNIVERSITY OF TECHNOLOGY

\title{
Control-Oriented Modeling of All-Solid-State Batteries Using Physics-Based Equivalent Circuits
}

Downloaded from: https://research.chalmers.se, 2023-04-26 10:42 UTC

Citation for the original published paper (version of record):

Li, Y., Wik, T., Xie, C. et al (2022). Control-Oriented Modeling of All-Solid-State Batteries Using Physics-Based Equivalent Circuits. IEEE Transactions on Transportation Electrification, 8(2): 2080-2092. http://dx.doi.org/10.1109/TTE.2021.3131147

N.B. When citing this work, cite the original published paper.

(C2022 IEEE. Personal use of this material is permitted.

However, permission to reprint/republish this material for advertising or promotional purposes 


\title{
Control-Oriented Modeling of All-Solid-State Batteries Using Physics-Based Equivalent Circuits
}

\author{
Yang Li, Member, IEEE, Torsten Wik, Changjun Xie, Member, IEEE, Yicun Huang, \\ Binyu Xiong, Member, IEEE, Jinrui Tang, Member, IEEE, and Changfu Zou, Member, IEEE
}

\begin{abstract}
Considered as one of the ultimate energy storage technologies for electrified transportation, the emerging all-solidstate batteries (ASSBs) have attracted immense attention due to their superior thermal stability, increased power and energy densities, and prolonged cycle life. To achieve the expected high performance, practical applications of ASSBs require accurate and computationally efficient models for the design and implementation of many onboard management algorithms, so that the ASSB safety, health, and cycling performance can be optimized under a wide range of operating conditions. A controloriented modeling framework is thus established in this work by systematically simplifying a rigorous partial differential equation (PDE) based model of the ASSBs developed from underlying electrochemical principles. Specifically, partial fraction expansion and moment matching are used to obtain ordinary differential equation based reduced-order models (ROMs). By expressing the models in a canonical circuit form, excellent properties for control design such as structural simplicity and full observability are revealed. Compared to the original PDE model, the developed ROMs have demonstrated high fidelity at significantly improved computational efficiency. Extensive comparisons have also been conducted to verify its superiority to the prevailing models due to the consideration of concentration-dependent diffusion and migration. Such ROMs can thus be used for advanced control design in future intelligent management systems of ASSBs.
\end{abstract}

Index Terms-All-solid-state battery, battery modeling, electrochemical model, model order reduction, moment matching.

\section{INTRODUCTION}

$\mathbf{R}$ ECHARGEABLE batteries have become ubiquitous energy storage devices in recent years for use in both mobile applications, including electric vehicles (EVs) and consumer devices, as well as stationary applications such as to mitigate the impact of fluctuating renewable generation to grid systems in the form of large-scale battery energy storage systems [1], [2]. These modern applications require safe, reliable, and efficient operation under a wide range of operating conditions, currently being dominated by lithiumion (Li-ion) batteries. Li-ion batteries are experiencing rapid development as one of the most important energy storage technologies due to their relatively high cell voltage, low self-discharge, and an excellent tradeoff between power and

This work was supported by EU-funded Marie Skłodowska-Curie Actions Individual Fellowships under Grant 895337-BatCon-H2020-MSCA-IF-2019.

Yang Li, Torsten Wik, Yicun Huang, and Changfu Zou are with the Department of Electrical Engineering, Chalmers University of Technology, 41296 Gothenburg, Sweden (e-mail: yangli@ieee.org; tw@chalmers.se; yicun@chalmers.se; changfu.zou@chalmers.se).

Changjun Xie, Binyu Xiong, and Jinrui Tang are with the School of Automation, Wuhan University of Technology, Wuhan, Hubei 430070, China. (email: jackxie@whut.edu.cn; bxiong2@whut.edu.cn; tangjinrui@whut.edu.cn). energy densities [3]. However, conventional Li-ion batteries use organic electrolytes and porous electrodes, which poses several challenges in battery safety and cycling performance. First, the organic electrolytes, most of which are in liquid form, are highly flammable and can cause fire hazards/explosions during faulty conditions due to thermal runaway. Second, the organic liquid electrolyte usually has low conductivity, which places intrinsic technical limits on modern high-power applications such as fast charging for EVs. Furthermore, the porous electrodes have almost reached their theoretical power density limits, which makes the further increase of the power/energy density in EVs difficult [4].

All-solid-state batteries (ASSBs) have thus received increasing research attention in the last decade to sidestep the abovementioned problems of the conventional Li-ion batteries [5]. In ASSBs, the organic liquid or polymer electrolytes are replaced with new types of inorganic electrolytes in solid form, such as oxides with garnet structures, perovskites, and sodium superionic conductors. The solid electrolytes are nonflammable and highly thermal resistive, so to prevent the problems of electrolyte leakage, internal short circuit, and thermal runaway. In addition, the solid-state electrolytes have better compatibility with metallic $\mathrm{Li}$ anodes by providing a mechanical barrier to dendrite formation [6]. Also, the better adaptability to high-voltage cathode materials and metallic $\mathrm{Li}$ anodes will greatly increase the energy density of batteries. Such a type of battery is believed to have the merits of longer life, ensured safety, higher power and energy density, and easier to be packed towards minimization and flexibility for future applications [7].

Although there have been significant material and structural breakthroughs in ASSBs, an ASSB system requires to be properly monitored and controlled during practical operation to fulfill the expectation of its longevity and high performance. The information on the internal states can be used for establishing the charging/discharging strategies for the ASSB to balance the requirements of high safety, long service life, and fast load response. A suitable ASSB model with high fidelity and low computational burden for online operation is essential for most model-based management algorithms. A general approach to battery modeling is to use equivalent circuit models (ECMs) [8]. These have the advantage of ready implementation in well-accepted circuit simulation and control system design software packages such as MATLAB/Simulink. In these packages, various numerical solvers have been incorporated and can be selected to solve the circuit model to facilitate the design of the control system. Conventionally 
obtained from system identification, the parametric values of such empirical models shall be adjusted regularly to fit the measurement data from tests carried out on-site [9]. However, this approach to battery modeling has limited applicability for wider operating ranges and long-term battery performance prediction under the ever-changing system dynamics. Battery degradation and internal safety cannot be properly addressed due to the lack of mechanistic insight regarding electrochemical dynamics. Furthermore, modern applications of batteries need to be designed for higher current rates, increased load dynamics, and harsher operating environments. Under these circumstances, model order, function complexity, and workload for tests to identify parameters of ECM have to be drastically increased to achieve sufficient extrapolability. To address these shortcomings, the physics-based models with the capability of describing the internal electrochemical behaviors of $\mathrm{Li}$-ion batteries under a wide range of operating conditions have been developed [10]. For example, various electrochemical models have been established to describe the growth of solid-electrolyte interphase film and the lithium deposition that lead to the increase of internal resistance and the loss of battery capacity of the conventional Li-ion batteries [11]-[13], while it is difficult to design a health-aware charging strategy with empirically-derived ECMs. Proper reduced-order models (ROMs) have been developed for various applications in the emerging health- and safety-aware battery management systems for online operation since the physical limitation can be better predicted than the ECM-based algorithm [14].

Health- and safety-aware battery management are also achievable if relevant information can be accurately described for ASSBs, such as the lithium plating during the charging process [15] and stress-induced damage that can lead to mechanical degradation [16]. Since the physical structure of ASSBs is fundamentally different from the conventional liquid-electrolyte-based $\mathrm{Li}$-ion batteries, attempts have been made to establish rigorous models of ASSBs. Becker et al. [17] presented a model for ASSBs that takes into account detailed aspects of ion transport in solid solutions of crystalline metal oxides. More precisely, the diffuse part of the double layer is dynamically described via the Poisson equation, while the Stern layer potential drop is modeled by a Robin boundary condition. Electrochemical reactions on the electrode/electrolyte interface (EEI) are modeled via non-linear Neumann boundary conditions. After a detailed derivation of the model equations and boundary conditions, numerical results are presented and discussed. Danilov et al. [18] developed an isothermal ASSB model that considered the imperfect dissociation of the ions in the electrolyte. This model consists of two partial differential equations (PDEs) that describe the diffusion processes in the solid electrolyte and in the positive electrode. In view of this, model simplification is of significant importance for many real-world applications such as charging strategy evaluation, residual capacity/lifetime estimation, thermal management, cell balancing, and fast charging control. Battery management algorithms such as state estimation have been developed recently based on this model [19], where the PDEs are solved using the finite difference method. However, this method shall create a high system order and the efficiency of the algorithm has not been studied. In [20], a rigorous PDE model has been reduced using a combination of Padé approximation, polynomial profile approximation, and equalreaction-coefficient assumption, and the computational burden was significantly reduced with high fidelity amenable for online parameter estimation and state monitoring [21].

It is noticed that the PDE model [18] for developing the ROMs in [19]-[21] was established for specific thinfilm ASSBs where many internal phenomena can be ignored. However, most recent works [22], [23] have demonstrated the importance of considering the effects of concentrationdependent diffusion coefficient and ionic migration behavior in the positive electrode, which can significantly influence the charging/discharging capabilities of new ASSBs with wide positive electrodes. For example, it shows in [18] that a thinfilm ASSB cell with a $0.32 \mu \mathrm{m}$ positive electrode can readily sustain 51C constant current discharge, whereas in [23], an ASSB with a much wider electrode $(8.08 \mu \mathrm{m})$ is shown to be only suitable for up to $4 \mathrm{C}-6 \mathrm{C}$ discharge current rate, in which condition significant nonuniformity can be observed in the ionic diffusion properties in the positive electrode.

In the present investigation, aiming at increasing the applicability of the physics-based ASSB model for control purposes, we establish a framework of model order reduction for ASSBs by simplifying a high-fidelity PDE-based model with the consideration of concentration-dependent diffusion and migration in the positive electrode. Consequently, the major contribution in this paper is the development of this model simplification framework for ASSBs, first expressed in a form of standard state-space representation and reformed into an physics-based equivalent circuit. The procedure is systematically presented by using partial fraction expansion and moment matching (PFE-MM). The proposed method for model reduction is simple-to-derive and it removes the limitations on electrode width. The assumptions imposed on the system's physical and dynamic characteristics are quantitatively justified by simulation and compared with the experimentally-verified PDE model in the literature. The simplified models are not only well suited for analyzing the charging/discharging behaviors of ASSBs but also beneficial for the development of modelbased controllers and optimization algorithms for ASSBs.

\section{An Electrochemical Model of ASSBs}

This section presents an overview of the high-fidelity PDE model of the ASSBs under investigation. The purpose is to provide a fundamental background of the ASSB structure and relevant governing equations for the development of the model simplification framework in latter sections. If not specifically indicated, all the equations are based on the benchmark PDE model developed in [23].

\section{A. Description of ASSBS}

A schematic of the one-dimensional model of a typical ASSB cell is shown in Fig. 1. The cell is divided into three physical domains along the horizontal axis, including the porous positive electrode (denoted by " $n$ "), the negative electrode (a metallic Li foil, denoted by " $p$ "), and the solid 


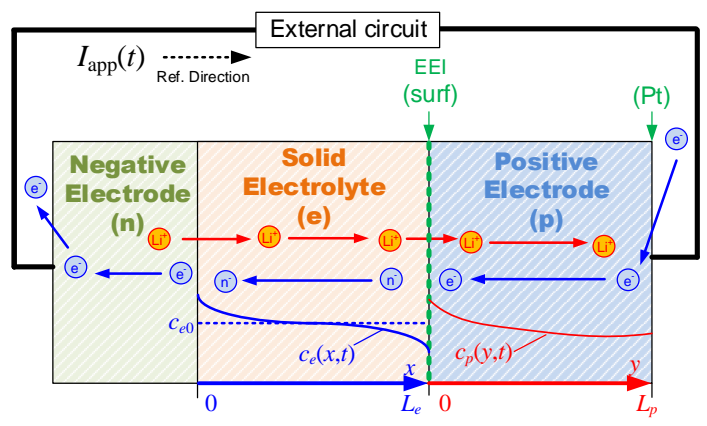

Fig. 1. Schematic of an ASSB cell during a discharge process. $\mathrm{Li}^{+}$: lithium ion. $e^{-}$: electron. $n^{-}$: uncompensated negative charge in electrolyte.

electrolyte compartment in between (denoted by " $e$ "). As shown in Fig. 1, the inner and the outer physical boundaries of the positive electrode domain are denoted by $y=0$ and $y=L_{p}$, respectively, where $L_{p}$ is the width of the positive electrode. The outer boundary of the positive electrode is attached to a platinum $(\mathrm{Pt})$ current collector. For the ease of notation, superscripts "surf" and "Pt" are used next to denote the local quantities at the boundary $y=0$ and $y=L_{p}$, respectively, i.e., for a spatiotemporal variable or parameter $X(y, t)$ in the positive electrode domain, $X^{\text {surf }}:=X(y=0, t)$ and , $X^{\mathrm{Pt}}:=X\left(y=L_{p}, t\right)$. The two boundaries of the solid electrolyte domain are denoted by $x=0$ and $x=L_{e}$, respectively, with $L_{e}$ being the width of the solid electrolyte. The functionalities of the solid electrolyte are two-fold: it allows the ionic conduction between the two electrodes, while it separates the electrons and forces them to move via the external circuit. Different from the conventional Li-ion batteries in which the porous electrodes are immersed in liquid or polymer electrolyte, here, the solid positive electrode is attached to the solid electrolyte, forming a solid-solid contact at the EEI $\left(x=L_{e}\right.$ or $\left.y=0\right)$. The $\mathrm{Li}$ ions will transport from the negative electrode to the positive electrode via the solid electrolyte by means of diffusion and migration during the discharge process, and a reverse process occurs when the battery is being charged. The current $I_{\text {app }}$ (defined as positive during charging) flows between the Pt current collector and the metallic $\mathrm{Li}$ electrode through the external circuit due to the movement of electrons. This structure is much simpler than the conventional Li-ion batteries, which facilitates the package for manufacturing [24]. Note that the double-layer and geometrical capacitances are not discussed in this work since they have ignorable effects on battery performance at medium and low-frequency $(<1 \mathrm{~Hz})$ regions under investigation for common battery management functionalities.

\section{B. Diffusion in the Solid Electrolyte}

In the solid electrolyte, the mobile Li ions exist in equilibrium with the immobile $\mathrm{Li}$ ions. The immobile $\mathrm{Li}$ ions can undergo an ionization reaction to generate the mobile Li ions and uncompensated negative charges during a charge process, and a reverse reaction occurs during discharging. Under the electroneutrality condition, the diffusion and migration of the mobile Li ions can be described by

$$
\frac{\partial c_{e}(x, t)}{\partial t}=D_{e}^{\mathrm{eff}} \frac{\partial^{2} c_{e}(x, t)}{\partial x^{2}}+r
$$

where $c_{e}$ is the concentration of the mobile $\mathrm{Li}$ ions in the solid electrolyte, $D_{e}^{\mathrm{eff}}:=2 D_{e}^{+} D_{e}^{-} /\left(D_{e}^{+}+D_{e}^{-}\right)$is the effective binary diffusion coefficient in the electrolyte, and $D_{e}^{+}$and $D_{e}^{-}$are the diffusion coefficients of the mobile $\mathrm{Li}$ ions and the uncompensated negative charges in the electrolyte, respectively. Note that the diffusion coefficients in the electrolyte are considered independent of the electrolyte concentration $c_{e}$ since the variation of electrolyte concentration is not significant, and within a narrow range of concentration the diffusion coefficients can be considered to be constant. Furthermore, $r$ is a charge carrier generation term, considering ignorable in existing works [19], [20]. The boundary conditions and the initial condition of (1a) are given by

$$
\begin{gathered}
\left.D_{e}^{+} \frac{\partial c_{e}(x, t)}{\partial x}\right|_{x=0}=\left.D_{e}^{+} \frac{\partial c_{e}(x, t)}{\partial x}\right|_{x=L_{e}}=\frac{i_{\text {app }}(t)}{2 F} \\
c_{e}(x, t=0)=c_{e 0}=\delta c_{0}
\end{gathered}
$$

where $F$ is the Faraday constant and $i_{\text {app }}=I_{\text {app }} / A$ is the applied current density with $A$ being the cross-sectional area of the cell. In addition, $c_{e 0}$ and $c_{0}$ represent the initial concentration of the mobile $\mathrm{Li}$ ions and the total $\mathrm{Li}$ ions in the electrolyte, respectively, and $\delta$ is the fraction of Li ions in the mobile states under the equilibrium condition.

\section{Mass-Transfer Overpotential of the Solid Electrolyte}

Diffusion and migration in the solid electrolyte induce the mass-transfer overpotential $\eta_{e}^{\mathrm{mt}}$, which is expressed by

$$
\eta_{e}^{\mathrm{mt}}(t)=\frac{R T}{F} \ln \left(\frac{c_{e}\left(L_{e}, t\right)}{c_{e}(0, t)}\right)-\int_{0}^{L_{e}} E_{e}(x, t) d x
$$

where the first and the second terms on the RHS are due to diffusion and migration, respectively. Here, $R$ is the universal gas constant, $T$ is the cell temperature, and $E_{e}$ is the electric field in the electrolyte given by

$$
\begin{aligned}
& E_{e}(x, t)=\frac{R T}{F} \frac{1}{c_{e}(x, t)} \\
& \times\left[\frac{-i_{\mathrm{app}}(t)}{2 F D_{e}^{+}}+\frac{D_{e}^{+}-D_{e}^{-}}{D_{e}^{+}+D_{e}^{-}}\left(\frac{\partial c_{e}(x, t)}{\partial x}+\frac{-i_{\mathrm{app}}(t)}{2 F D_{e}^{+}}\right)\right] .
\end{aligned}
$$

The reader is referred to [25] for details of the derivation and more explanations of (2) and (3).

\section{Concentration-Dependent Diffusion of Positive Electrode}

Assuming the effect of phase transition can be ignored and considering the limited diffusion rate of electrons in the positive electrode of an ASSB, the diffusion of $\mathrm{Li}$ ions in the positive electrode can be described by,

$$
\frac{\partial c_{p}(y, t)}{\partial t}=\frac{\partial}{\partial y}\left(D_{p}^{\mathrm{eff}} \frac{\partial c_{p}(y, t)}{\partial y}\right)
$$


with the boundary and initial conditions

$$
\begin{gathered}
\left.D_{p}^{+} \frac{\partial c_{p}(y, t)}{\partial y}\right|_{y=0}=-\left.D_{p}^{-} \frac{\partial c_{p}(y, t)}{\partial y}\right|_{y=L_{p}}=\frac{i_{\text {app }}(t)}{2 F} \\
c_{p}(y, t=0)=c_{p 0}
\end{gathered}
$$

where $c_{p}$ is the concentration of the $\mathrm{Li}$ ions in the positive electrode, $D_{p}^{\text {eff }}:=2 D_{p}^{+} D_{p}^{-} /\left(D_{p}^{+}+D_{p}^{-}\right)$is the effective binary diffusion coefficient in the positive electrode, and $D_{p}^{+}$and $D_{p}^{-}$ are the diffusion coefficients of $\mathrm{Li}$ ions and the electrons in the positive electrode, respectively. In contrast to the electrolyte, the concentration in the positive electrode can vary within a much wider range. Hence, $D_{p}^{+}$and $D_{p}^{-}$are considered concentration-dependent, denoted by $D_{p}^{+}\left(c_{p}\right)=f_{D+}\left(c_{p}\right)$ and $D_{p}^{-}\left(c_{p}\right)=f_{D-}\left(c_{p}\right)$, respectively.

The state-of-charge (SOC) of the ASSB is determined by the volumed-average concentration $c_{p}^{\text {avg }}$, i.e.,

$$
\operatorname{SOC}(t)=\frac{c_{p}^{100 \%}-c_{p}^{\mathrm{avg}}(t)}{c_{p}^{100 \%}-c_{p}^{0 \%}}=\frac{c_{p}^{100 \%}-\frac{1}{L_{p}} \int_{0}^{L_{p}} c_{p}(y, t) d y}{c_{p}^{100 \%}-c_{p}^{0 \%}},
$$

where $c_{p}^{100 \%}$ and $c_{p}^{0 \%}$ are the concentrations of $\mathrm{Li}$ ions correspond to SOC $=100 \%$ and SOC $=0 \%$, respectively.

\section{E. Equilibrium Potential and Mass-Transfer Overpotential of the Electrodes}

Concentrations $c_{p}^{\text {surf }}(t):=c_{p}(0, t)$ and $c_{p}^{\mathrm{Pt}}(t):=c_{p}\left(L_{p}, t\right)$ at the two boundaries of the positive electrode are needed to calculate the positive electrode potential $U_{p}=U_{p}^{\mathrm{eq}}+\eta_{p}^{\mathrm{mt}}$, consisting of the thermodynamic equilibrium potential $U_{p}^{\mathrm{eq}}$ and the overpotential $\eta_{p}^{\mathrm{mt}}$ due to mass transfer in the electrode, i.e.,

$$
\begin{gathered}
U_{p}^{\mathrm{eq}}(t)=h_{1}\left(c_{p}^{\mathrm{surf}}(t)\right) \\
\eta_{p}^{\mathrm{mt}}(t)=\frac{R T}{F} \ln \left(\frac{c_{p}^{\mathrm{Pt}}(t)}{c_{p}^{\text {surf }}(t)}\right)-\int_{0}^{L_{p}} E_{p}(y, t) d y,
\end{gathered}
$$

where $h_{1}(\cdot)$ is a nonlinear function determined by the materials of the positive electrode. Similar to (2), the first and the second terms on the RHS of (7) are due to diffusion and migration in the positive electrode, respectively, while the electric field $E_{p}$ is given by

$$
\begin{aligned}
& E_{p}(y, t)=\frac{R T}{F} \frac{1}{c_{p}(y, t)} \\
& \times\left[\frac{-i_{\mathrm{app}}(t)}{2 F D_{p}^{+}}+\frac{D_{p}^{+}-D_{p}^{-}}{D_{p}^{+}+D_{p}^{-}}\left(\frac{\partial c_{p}(y, t)}{\partial y}+\frac{-i_{\mathrm{app}}(t)}{2 F D_{p}^{+}}\right)\right] .
\end{aligned}
$$

Note that the overpotential $\eta_{p}^{\mathrm{mt}}$ cannot be ignored unless the diffusion process of electrons is much faster than that of the $\mathrm{Li}$ ions. The reader is referred to [23] for a detailed discussion on the effect of the diffusion coefficients in the positive electrode.

Furthermore, since the negative electrode is a metallic $\mathrm{Li}$ foil, the Li concentration $c_{n}$ in this electrode is considered a constant and equal to the average bulk concentration of the negative electrode $c_{n}^{\text {avg }}$. The potential of the negative electrode $U_{n}$ is assumed to be zero due to the high conductivity of the Li metal [22], [23].

\section{F. Charge-Transfer Overpotentials}

The intercalation/de-intercalation reaction kinetics are described by the Butler-Volmer equation and the charge-transfer coefficients for both electrodes are assumed to be 0.5 in this work [19]. The expressions of the charge-transfer overpotential for both electrodes are thus given by

$$
\begin{aligned}
& \eta_{p}^{\mathrm{ct}}(t)=\frac{2 R T}{F} \sinh ^{-1}\left(\frac{i_{\text {app }}(t)}{2 i_{0, p}(t)}\right) \\
& \eta_{n}^{\mathrm{ct}}(t)=\frac{2 R T}{F} \sinh ^{-1}\left(\frac{-i_{\text {app }}(t)}{2 i_{0, n}(t)}\right)
\end{aligned}
$$

where $i_{0}$ represents the average exchange current density for intercalation defined under equilibrium conditions, given by

$$
\begin{aligned}
& i_{0, p}(t)=F k_{0, p}\left[c_{e 0} c_{p}^{\text {surf }}(t)\right]^{0.5}\left[c_{p}^{\text {max }}-c_{p}^{\text {surf }}(t)\right]^{0.5} \\
& i_{0, n}(t)=F k_{0, n}\left(c_{e 0}\right)^{0.5}\left(c_{n}^{\text {avg }}\right)^{0.5}
\end{aligned}
$$

where $c_{p}^{\max }$ is the theoretical maximum concentration in the positive electrode. $k_{0, p}$ and $k_{0, n}$ are the reaction constants of the two electrodes.

\section{G. Terminal Voltage}

The ASSB voltage is the sum of the equilibrium potential of the positive electrode and various overpotentials, i.e.,

$$
V_{\text {bat }}(t)=U_{p}^{\mathrm{eq}}(t)+\eta_{p}^{\mathrm{mt}}(t)+\eta_{e}^{\mathrm{mt}}(t)+\eta_{p}^{\mathrm{ct}}(t)-\eta_{n}^{\mathrm{ct}}(t),
$$

where the contact resistance at the EEI and the current collector resistance are ignored.

\section{A Model Reduction Framework for ASSBs}

The PDE-based ASSB model (1)-(11) is considered accurate but difficult for both control system design and online implementation. For the purpose of overcoming this, in this section, a model order reduction framework is established.

\section{A. Simplified Solid Electrolyte Diffusion Equation}

Defining $t_{e}^{+}:=D_{e}^{+} /\left(D_{e}^{+}+D_{e}^{-}\right)$as the transference number of the electrolyte and $t_{e}^{-}:=D_{e}^{-} /\left(D_{e}^{+}+D_{e}^{-}\right)=1-t_{e}^{+}$, the boundary conditions $(1 \mathrm{~b})$ can be reformulated into

$$
\left.D_{e}^{\mathrm{eff}} \frac{\partial c_{e}(x, t)}{\partial x}\right|_{x=0}=\left.D_{e}^{\mathrm{eff}} \frac{\partial c_{e}(x, t)}{\partial x}\right|_{x=L_{e}}=\frac{t_{e}^{-}}{F} i_{\mathrm{app}}(t) .
$$

Taking the Laplace transform of (1) with the boundary conditions (12), one can obtain the following transcendental transfer function,

$$
\frac{c_{e}(x, s)}{i_{\mathrm{app}}(s)}=-\frac{t_{e}^{-} \tau_{e}}{2 F L_{e}} \frac{1}{\frac{1}{2} \sqrt{\tau_{e} s}} \frac{\sinh \left(\left(\frac{2 x}{L_{e}}-1\right) \cdot \frac{1}{2} \sqrt{\tau_{e} s}\right)}{\cosh \left(\frac{1}{2} \sqrt{\tau_{e} s}\right)},
$$

where $\tau_{e}=L_{e}^{2} / D_{e}^{\text {eff }}$ is the time constant for the electrolyte diffusion. The derivation is detailed in the Appendix.

Note that (13) has an anti-symmetrical property about the middle point $x=L_{e} / 2$ of the solid-electrolyte domain, rendering $c_{e}(x, s) / i_{\text {app }}(s)=-c_{e}\left(L_{e}-x, s\right) / i_{\text {app }}(s)$, and this gives $c_{e}(x, t)+c_{e}\left(L_{e}-x, t\right)=2 c_{e 0}$ in the time domain. As seen in (2), we are particularly interested in the electrolyte concentrations at the domain boundaries. Hence, denoting the 
TABLE I

PFE COEFFICIENTS FOR ELECTROLYTE DIFFUSION EQUATION

\begin{tabular}{ccccccccccc}
\hline \hline & $a_{e, 1}$ & $b_{e, 1}$ & $a_{e, 2}$ & $b_{e, 2}$ & $a_{e, 3}$ & $b_{e, 3}$ & $a_{e, 4}$ & $b_{e, 4}$ & $a_{e, 5}$ & $b_{e, 5}$ \\
\hline$N_{e}=1$ & 12 & 12 & - & - & - & - & - & - & - & - \\
$N_{e}=2$ & 9.88 & 8.02 & 170.12 & 31.98 & - & - & - & - & - & - \\
$N_{e}=3$ & 9.87 & 8.00 & 91.22 & 9.01 & 738.9 & 66.99 & - & - & - & - \\
$N_{e}=4$ & 9.87 & 8.00 & 88.86 & 8.02 & 275.77 & 12.5 & 2145.5 & 115.48 & - & - \\
$N_{e}=5$ & 9.87 & 8.00 & 88.83 & 8.00 & 248.45 & 8.39 & 618.84 & 18.57 & 4974.02 & 177.04 \\
\hline \hline
\end{tabular}

concentration at the EEI $c_{e}^{\text {surf }}(t):=c_{e}\left(L_{e}, t\right)$ and using (13), the following transcendental transfer function can be obtained:

$$
\frac{c_{e}^{\text {surf }}(s)}{i_{\text {app }}(s)}=-\frac{t_{e}^{-} \tau_{e}}{2 F L_{e}} G_{e}(s)
$$

where

$$
G_{e}(s)=\frac{\tanh \left(\frac{1}{2} \sqrt{\tau_{e} s}\right)}{\frac{1}{2} \sqrt{\tau_{e} s}}
$$

The transcendental transfer function (14) represents an infinite-dimensional system. For the purpose of control system design and implementation, a low-order rational transfer function describing a practically realizable system is needed. Since all singularities of $G_{e}(s)$ are of first order [26], and located on the negative real axis, it can be approximated by a series of first order transfer functions, i.e., we may assume a partial fraction expansion (PFE),

$$
G_{e}(s) \approx P_{e}(s)=\sum_{i=1}^{N_{e}} \frac{b_{e, i}}{\tau_{e} s+a_{e, i}},
$$

where $N_{e}$ is the order of the approximation and $a_{e, i}$ and $b_{e, i}$ are the PFE coefficients for the electrolyte diffusion equation. With this approximation, the transfer function (16) can be readily realized as

$$
\begin{aligned}
\frac{d \tilde{c}_{e, i}(t)}{d t} & =-\frac{a_{e, i}}{\tau_{e}} \tilde{c}_{e, i}(t)-\frac{b_{e, i} t_{e}^{-}}{2 F L_{e}} i_{\mathrm{app}}(t) \quad \forall i \in \mathcal{N}_{e} \\
c_{e}^{\mathrm{surf}}(t) & =c_{e 0}+\sum_{i=1}^{N_{e}} \tilde{c}_{e, i}(t)
\end{aligned}
$$

where $\mathcal{N}_{e}=\left\{1,2, \cdots, N_{e}\right\}$.

To determine the $2 N_{e}$ PFE coefficients $a_{e, i}$ and $b_{e, i}, i \in \mathcal{N}_{e}$, moment matching (MM) is adopted here [27]. Specifically, we set $P_{e}(0)=G_{e}(0)$ and do the same for the first $\left(2 N_{e}-1\right)$ derivatives of $P_{e}(0)=G_{e}(0)$ for $s=0$, establishing $2 N_{e}$ equations that uniquely determines the values of all coefficients $a_{e, i}$ and $b_{e, i}$. The results up to $N_{e}=5$ are provided in Table I. In Fig. 2, Bode diagrams of the proposed ROMs of (13) are compared with several other methods, using the parameters given in Section IV, including the ideal PDE model, the FVM, and an analytical method to determine the coefficients $a_{e, i}$ and $b_{e, i}$ based on the residue theory [26]. It can be seen that the frequency-domain approximation with the proposed method generally outperforms the other methods: With the same order, the proposed ROM is more accurate than the ROM based on residue theory, and a 5th-order proposed ROM is comparable to 100 th-order FVM model.
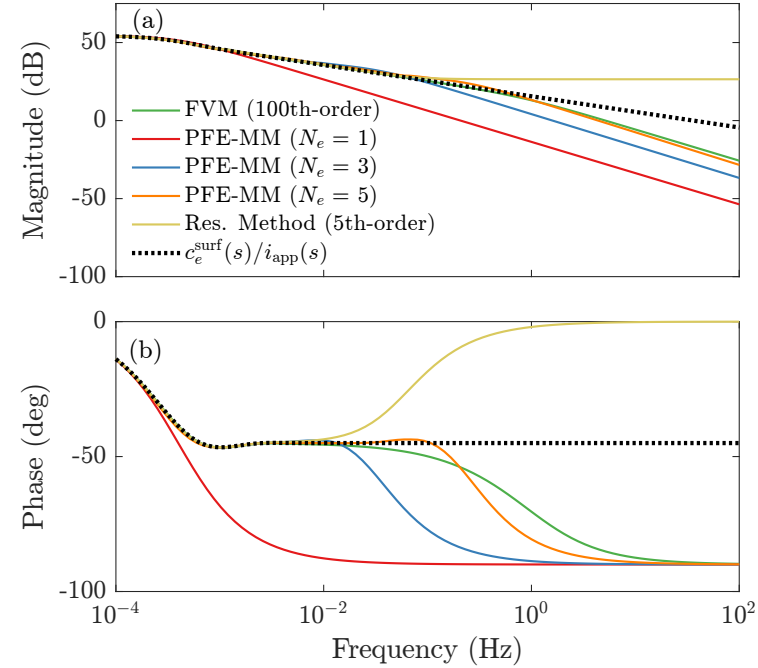

Fig. 2. Bode diagram for the solid electrolyte diffusion equation at $x=L_{e}$ using different model order reduction methods. (a) Magnitude response. (b) Phase response.

\section{B. Simplified Positive Electrode Diffusion Equation Under Constant Diffusion Coefficients}

Similar to the solid electrolyte, the boundary conditions (4b) of the diffusion equation (4a) of the positive electrode can be rewritten as

$$
\left.\frac{D_{p}^{\mathrm{eff}}}{t_{p}^{-}} \frac{\partial c_{p}(y, t)}{\partial y}\right|_{y=0}=-\left.\frac{D_{p}^{\mathrm{eff}}}{t_{p}^{+}} \frac{\partial c_{p}(y, t)}{\partial y}\right|_{y=L_{p}}=\frac{1}{F} i_{\text {app }}(t),
$$

where $t_{p}^{+}:=D_{p}^{+} /\left(D_{p}^{+}+D_{p}^{-}\right)$is the transference number of the positive electrode and $t_{p}^{-}=1-t_{p}^{+}$. Note that $D_{p}^{+}, D_{p}^{-}, D_{p}^{\text {eff }}$, $t_{p}^{+}$, and $t_{p}^{-}$are all concentration-dependent and nonuniform in the positive electrode domain. In order to obtain a transfer function, we assume the values of these parameters are equal at the two boundaries, e.g., $D_{p}^{+ \text {,surf }}=D_{p}^{+, \text {Pt }}, D_{p}^{- \text {,surf }}=D_{p}^{-, \text {Pt }}$, etc. Applied to (18), a transcendental transfer function for (4a) can then be obtained by applying the Laplace transform, i.e.,

$$
\begin{aligned}
\frac{c_{p}(y, s)}{i_{\text {app }}(s)} & =-\frac{1}{F L_{p} s}-\frac{\tau_{p}}{F L_{p}} \\
& \times\left(\frac{1}{\sqrt{\tau_{p} s}} \frac{\cosh \left(\left(1-\frac{y}{L_{p}}\right) \sqrt{\tau_{p} s}\right)}{\sinh \left(\sqrt{\tau_{p} s}\right)}-\frac{1}{\tau_{p} s}\right) \\
& -\frac{t_{p}^{+} \tau_{p}}{2 F L_{p}} \frac{1}{\frac{1}{2} \sqrt{\tau_{p} s}} \frac{\sinh \left(\left(\frac{2 y}{L_{p}}-1\right) \frac{1}{2} \sqrt{\tau_{p} s}\right)}{\cosh \left(\frac{1}{2} \sqrt{\tau_{p} s}\right)},
\end{aligned}
$$


where $\tau_{p}=L_{p}^{2} / D_{p}^{\text {eff }}$ is the time constant for the diffusion process in the positive electrode. According to (6) and (7), the positions of interest are the boundaries $y=0$ and $y=L_{p}$. Unlike the electrolyte diffusion equation (1), there is no general (anti-)symmetrical relationships in the positive electrode domain, and thus the transfer functions for these two positions have to be approximated individually. The procedures are described as follows.

At $y=0$, we have

$$
\frac{c_{p}^{\text {surf }}(s)}{i_{\text {app }}(s)}=-\frac{1}{F L_{p} s}-\frac{\tau_{p}^{\text {surf }}}{F L_{p}} G_{p}^{\text {surf }}(s)
$$

where

$$
\begin{aligned}
G_{p}^{\text {surf }}(s)= & \left(\frac{1}{\sqrt{\tau_{p}^{\text {surf }} s}} \frac{1}{\tanh \left(\sqrt{\tau_{p}^{\text {surf }} s}\right)}-\frac{1}{\tau_{p}^{\text {surf }} s}\right) \\
& -\frac{t_{p}^{+, \text {surf }}}{2} \frac{\tanh \left(\frac{1}{2} \sqrt{\tau_{p}^{\text {surf }} s}\right)}{\frac{1}{2} \sqrt{\tau_{p}^{\text {surf }} s}} .
\end{aligned}
$$

The first term on the RHS of (20) contributes to the change of bulk concentration, and the second term is associated with the overpotentials caused by uneven distribution of the concentration. Also, $G_{p}^{\text {surf }}(s)$ can be shown to have only countable first order singularities on the negative real axis. We can therefore adopt a similar PFE method as described in Section III-A, to convert $G_{p}^{\text {surf }}(s)$ to a rational form

$$
G_{p}^{\text {surf }}(s) \approx \sum_{i=1}^{N_{p 1}} \frac{b_{p 1, i}}{\tau_{p}^{\text {surf }} s+a_{p 1, i}}+\frac{t_{p}^{+, \text {surf }}}{2} \sum_{i=1}^{N_{p 2}} \frac{b_{p 2, i}}{\tau_{p}^{\text {surf }} s+a_{p 2, i}}
$$

Thus, (20) can be realized by

$$
\begin{aligned}
\frac{d c_{p}^{\text {avg }}(t)}{d t} & =-\frac{1}{F L_{p}} i_{\text {app }}(t) \\
\frac{d \tilde{c}_{p 1, i}(t)}{d t} & =-\frac{a_{p 1, i}}{\tau_{p}^{\text {surf }}} \tilde{c}_{p 1, i}(t)-\frac{b_{p 1, i}}{F L_{p}} i_{\text {app }}(t) \quad \forall i \in \mathcal{N}_{p 1} \\
\frac{d \tilde{c}_{p 2, i}(t)}{d t} & =-\frac{a_{p 2, i}}{\tau_{p}^{\text {surf }}} \tilde{c}_{p 2, i}(t)-\frac{t_{p}^{+, \text {surf }}}{2} \frac{b_{p 2, i}}{F L_{p}} i_{\text {app }}(t) \quad \forall i \in \mathcal{N}_{p 2} \\
c_{p}^{\text {surf }}(t) & =c_{p}^{\text {avg }}(t)+\sum_{i=1}^{N_{p 1}} \tilde{c}_{p 1, i}(t)+\sum_{i=1}^{N_{p 2}} \tilde{c}_{p 2, i}(t)
\end{aligned}
$$

where $\mathcal{N}_{p 1}=\left\{1, \cdots, N_{p 1}\right\}$ and $\mathcal{N}_{p 2}=\left\{1, \cdots, N_{p 2}\right\}$.

Similarly, at the other boundary $y=L_{p}$, the corresponding approximated transfer function can be derived by

$$
\frac{c_{p}^{\mathrm{Pt}}(s)}{i_{\text {app }}(s)}:=\frac{c_{p}\left(L_{p}, s\right)}{i_{\text {app }}(s)}=-\frac{1}{F L_{p} s}-\frac{\tau_{p}^{\mathrm{Pt}}}{F L_{p}} G_{p}^{\mathrm{Pt}}(s)
$$

where

$$
\begin{aligned}
G_{p}^{\mathrm{Pt}}(s) & =\left(\frac{1}{\sqrt{\tau_{p}^{\mathrm{Pt}}} s} \frac{1}{\sinh \left(\sqrt{\tau_{p}^{\mathrm{Pt}} s}\right)}-\frac{1}{\tau_{p}^{\mathrm{Pt}} s}\right) \\
& +\frac{t_{p}^{+}, \mathrm{Pt} \tanh \left(\frac{1}{2} \sqrt{\tau_{p}^{\mathrm{Pt}} s}\right)}{\frac{1}{2} \sqrt{\tau_{p}^{\mathrm{Pt}} s}}
\end{aligned}
$$
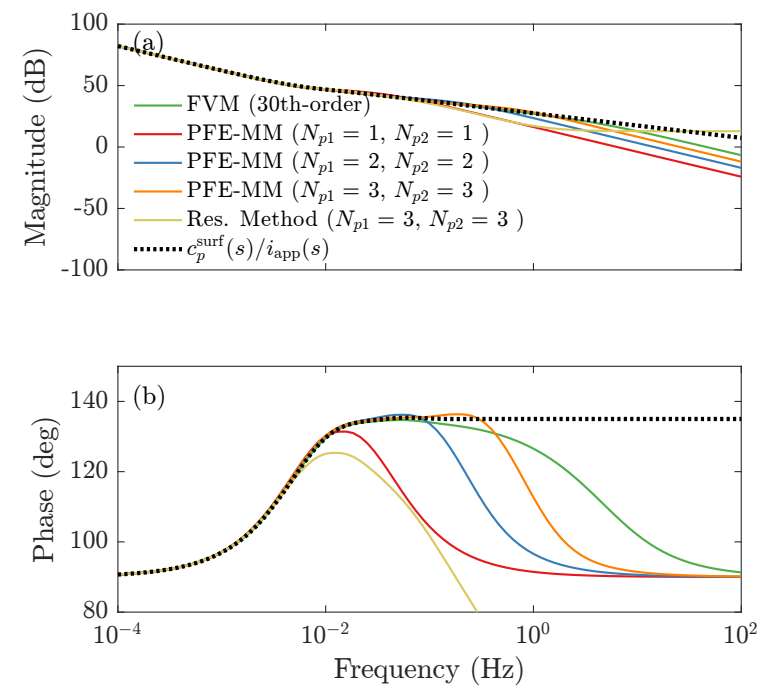

Fig. 3. Bode diagram for the positive electrode diffusion equation at $y=0$ using different order reduction methods. (a) Magnitude response. (b) Phase response.

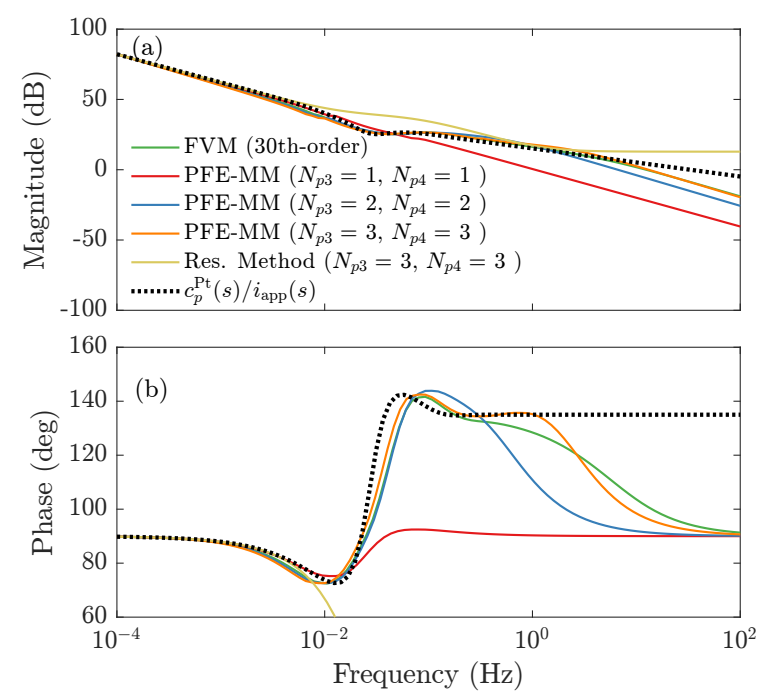

Fig. 4. Bode diagram for the positive electrode diffusion equation at $y=L_{p}$ using different order reduction methods. (a) Magnitude response. (b) Phase response.

$$
\approx \sum_{i=1}^{N_{p 3}} \frac{b_{p 3, i}}{\tau_{p}^{\mathrm{Pt}} s+a_{p 3, i}}+\frac{t_{p}^{+, \mathrm{Pt}}}{2} \sum_{i=1}^{N_{p 4}} \frac{b_{p 4, i}}{\tau_{p}^{\mathrm{Pt}} s+a_{p 4, i}} .
$$

The corresponding realization of (24) is

$$
\begin{aligned}
\frac{d \tilde{c}_{p 3, i}(t)}{d t} & =-\frac{a_{p 3, i}}{\tau_{p}^{\mathrm{Pt}}} \tilde{c}_{p 3, i}(t)-\frac{b_{p 3, i}}{F L_{p}} i_{\mathrm{app}}(t) \quad \forall i \in \mathcal{N}_{p 3} \\
\frac{d \tilde{c}_{p 4, i}(t)}{d t} & =-\frac{a_{p 4, i}}{\tau_{p}^{\mathrm{Pt}}} \tilde{c}_{p 4, i}(t)-\frac{t_{p}^{+, \mathrm{Pt}}}{2} \frac{b_{p 4, i}}{F L_{p}} i_{\mathrm{app}}(t) \quad \forall i \in \mathcal{N}_{p 4} \\
c_{p}^{\mathrm{Pt}}(t) & =c_{p}^{\mathrm{avg}}(t)+\sum_{i=1}^{N_{p 3}} \tilde{c}_{p 3, i}(t)+\sum_{i=1}^{N_{p 4}} \tilde{c}_{p 4, i}(t)
\end{aligned}
$$


TABLE II

PFE COEFFicients for Positive Electrode Diffusion EQUation

\begin{tabular}{|c|c|c|c|c|c|c|c|c|c|c|c|c|c|}
\hline & $a_{p 1,1}$ & $b_{p 1,1}$ & $a_{p 1,2}$ & $b_{p 1,2}$ & $a_{p 1,3}$ & $b_{p 1,3}$ & & $a_{p 3,1}$ & $b_{p 3,1}$ & $a_{p 3,2}$ & $b_{p 3,2}$ & $a_{p 3,3}$ & $b_{p 3,3}$ \\
\hline$N_{p 1}=1$ & 15 & 5 & - & - & - & - & $N_{p 3}=1$ & 8.57 & -1.43 & - & - & - & - \\
\hline$N_{p 1}=2$ & 9.94 & 2.07 & 95.06 & 11.93 & - & - & $N_{p 3}=2$ & 9.9 & -2.026 & 30.50 & 1.163 & - & - \\
\hline$N_{p 1}=3$ & 9.87 & 2 & 41.98 & 2.6 & 326.15 & 22.4 & $N_{p 3}=3$ & 9.87 & -2 & 41.2 & 2.68 & 59.08 & -1.718 \\
\hline
\end{tabular}

where $\mathcal{N}_{p 3}=\left\{1, \cdots, N_{p 3}\right\}$ and $\mathcal{N}_{p 4}=\left\{1, \cdots, N_{p 4}\right\}$.

Applying the MM method as described in the previous subsection, the PFE coefficients can be obtained. The PFE coefficients $a_{p 1, i}, b_{p 1, i}, a_{p 3, i}$, and $b_{p 3, i}$ up to $N_{p 1}=3$ and $N_{p 3}=3$ are given in Table II. Note that by comparing (21) and (25) with (15), we have $a_{p 2, i}=a_{p 4, i}=a_{e, i}$ and $b_{p 2, i}=-b_{p 4, i}=-b_{e, i}$, since they share the same transfer function up to a scaling of $s$.

Fig. 3 and Fig. 4 compare the Bode diagrams for the positive electrode diffusion equation with different order reduction methods at the two boundaries using the parameters given in Section IV. Again, it is shown the MM method is more accurate than the method based on residue theory and it can generate a much lower order system than the FVM to achieve a similar frequency response.

\section{Simplified Positive Electrode Diffusion Equation Under Concentration-Dependent Diffusion Coefficients}

Note that the results in Section III-B are only valid under the assumption of constant $D_{p}^{+}$and $D_{p}^{-}$. When $D_{p}^{+}$and $D_{p}^{-}$are weakly concentration-dependent, by updating the coefficients in each time step, the linear-time varying system can capture the dynamics. However, if the coefficients vary significantly, the linear approximated equations can lead to considerable accumulated error in the results over time. We therefore introduce a factor $\lambda$ to correct the time constant $\tau_{p}^{\text {surf }}$, i.e.,

$$
\tau_{p}^{\text {surf* }}=\lambda \tau_{p}^{\text {surf }}=\left(D_{p}^{+, \text {surf }} / D_{p}^{+, \text {Pt }}\right)^{k} \tau_{p}^{\text {surf }},
$$

where $k$ is a correction coefficient tuned by trial-and-error. It can be seen that $\lambda$ equals 1 either when the diffusion coefficients are uniform along the $y$ direction, or when $k=0$.

\section{Simplified Mass- and Charge-Transfer Overpotentials}

Equations (2) and (7) share the same form and they contain integrals. A numerical integration method is used to solve the equation [20], which increases the complexity of the model implementation and analysis. In this regard, we derive and use the following simplified expressions of the mass-transfer overpotentials, given by

$$
\begin{gathered}
\eta_{e}^{\mathrm{mt}}(t)=\frac{2 R T t_{e}^{-}}{F} \ln \left(\frac{c_{e}^{\text {surf }}(t)}{2 c_{e 0}-c_{e}^{\text {surf }}(t)}\right)+R_{e}^{\text {avg }} I_{\text {app }}(t) \\
:=h_{2}\left(c_{e}^{\text {surf }}(t)\right)+R_{e}^{\text {avg }} I_{\text {app }}(t) \\
\eta_{p}^{\mathrm{mt}}(t)=\frac{2 R T t_{p}^{-}}{F} \ln \left(\frac{c_{p}^{\mathrm{Pt}}(t)}{c_{p}^{\text {surf }}(t)}\right)+R_{p}^{\text {avg }} I_{\text {app }}(t) \\
:=h_{3}\left(c_{p}^{\text {surf }}(t), c_{p}^{\mathrm{Pt}}(t)\right)+R_{p}^{\text {avg }} I_{\text {app }}(t)
\end{gathered}
$$

where $R_{e}^{\mathrm{avg}}=L_{e} /\left[\left(D_{e}^{+}+D_{e}^{-}\right)\left(\frac{F^{2} A}{R T}\right) c_{e 0}\right]$ and $R_{p}^{\mathrm{avg}}=$ $L_{p} /\left[\left(D_{p}^{+}+D_{p}^{-}\right)\left(\frac{F^{2} A}{R T}\right) c_{p}^{\text {avg }}\right]$ are the volume-averaged resistances of the electrolyte and the positive electrode, respective1y. The detail of the derivation procedure is given in Appendix.

The charge-transfer overpotentials in (9) are rewritten by

$$
\begin{aligned}
& \eta_{p}^{\mathrm{ct}}(t)=R_{p}^{\mathrm{ct}} I_{\mathrm{app}}(t) \\
& \eta_{n}^{\mathrm{ct}}(t)=-R_{n}^{\mathrm{ct}} I_{\mathrm{app}}(t)
\end{aligned}
$$

where the charge-transfer resistances $R_{p}^{\mathrm{ct}}$ and $R_{n}^{\mathrm{ct}}$ are given by

$$
\begin{array}{r}
R_{p}^{\mathrm{ct}}= \begin{cases}\frac{R T}{F A i_{0, p}} \sinh \left(\frac{I_{\text {app }}}{2 A i_{0, p}}\right) /\left(\frac{I_{\text {app }}}{2 A i_{0, p}}\right), & I_{\text {app }} \neq 0 \\
\frac{R T}{F A i_{0, p}}, & i_{\text {app }}=0\end{cases} \\
R_{n}^{\mathrm{ct}}= \begin{cases}\frac{R T}{F A i_{0, n}} \sinh \left(\frac{I_{\text {app }}}{2 A i_{0, n}}\right) /\left(\frac{I_{\text {app }}}{2 A i_{0, n}}\right), & I_{\text {app }} \neq 0 \\
\frac{R T}{F A i_{0, n}}, & i_{\text {app }}=0\end{cases}
\end{array}
$$

\section{E. Summary of the Reduced-Order Model}

By now, we have obtained a ROM of ASSBs, and the model can be summarized in a compact continuous-time state-space form as follows:

$$
\begin{aligned}
\dot{\mathbf{x}}(t) & =f(\mathbf{x}(t), u(t))=\mathbf{A}(t) \mathbf{x}(t)+\mathbf{B}(t) u(t) \\
z(t) & =h(\mathbf{x}(t), u(t))
\end{aligned}
$$

where the input variable is the current $u=I_{\text {app}}$, the output variable is voltage $z=V_{\text {bat }}$, and the state vector is $\mathbf{x}=$ $\left[c_{p}^{\mathrm{avg}}, \tilde{\mathbf{c}}_{p 1}^{\top}, \tilde{\mathbf{c}}_{p 2}^{\top}, \tilde{\mathbf{c}}_{p 3}^{\top}, \tilde{\mathbf{c}}_{p 4}^{\top}, \tilde{\mathbf{c}}_{e}\right]^{\top} \in \mathbb{R}^{1+N_{p 1}+N_{p 2}+N_{p 3}+N_{p 4}+N_{e}}$, where $\quad \tilde{\mathbf{c}}_{p 1}=\left[\tilde{c}_{p 1,1}, \cdots, \tilde{c}_{p 1, N_{p 1}}\right]^{\top}, \quad \tilde{\mathbf{c}}_{p 2}=$ $\left[\tilde{c}_{p 2,1}, \cdots, \tilde{c}_{p 2, N_{p 2}}\right]^{\top}, \quad \tilde{\mathbf{c}}_{p 3}=\left[\tilde{c}_{p 3,1}, \cdots, \tilde{c}_{p 3, N_{p 3}}\right]^{\top}$, $\tilde{\mathbf{c}}_{p 4}=\left[\tilde{c}_{p 4,1}, \cdots, \tilde{c}_{p 4, N_{p 4}}\right]^{\top}$, and $\tilde{\mathbf{c}}_{e}=\left[\tilde{c}_{e, 1}, \cdots, \tilde{c}_{e, N_{e}}\right]^{\top}$. Note that the state equation (32a) is expressed in linear time-varying form, where the system matrix $\mathbf{A}$ and the input matrix $\mathbf{B}$ are given in (33) and (34). $\mathbf{A}$ and $\mathbf{B}$ are affected by the time constants and the transference numbers in the positive electrode, and these parameters are functions of the concentration-dependent diffusion coefficients.

On the other hand, the operator $h(\cdot)$ in the output equation (32b) is a nonlinear function of $c_{p}^{\text {surf }}(t), c_{p}^{\mathrm{Pt}}(t)$, and $c_{e}^{\text {surf }}(t)$, i.e.

$$
\begin{aligned}
h(\cdot) & =h_{1}\left(c_{p}^{\text {surf }}\right)+h_{2}\left(c_{e}^{\text {surf }}\right)+h_{3}\left(c_{p}^{\text {surf }}, c_{p}^{\mathrm{Pt}}\right) \\
& +\left(R_{e}^{\text {avg }}+R_{p}^{\text {avg }}+R_{p}^{\mathrm{ct}}+R_{n}^{\mathrm{ct}}\right) I_{\mathrm{app}}(t)
\end{aligned}
$$

with the nonlinear functions $h_{1}(\cdot), h_{2}(\cdot)$, and $h_{3}(\cdot)$ are provided in (6), (28), and (29), respectively, and $c_{p}^{\text {surf }}, c_{p}^{\mathrm{Pt}}$, and $c_{e}^{\text {surf }}$ are calculated using (17b), (23d), and (26c), respectively.

The derived ROM can readily be reformulated with the equivalent circuit representation as shown in Fig. 5, where the equivalent resistances and capacitances are expressed as the 


$$
\begin{gathered}
\mathbf{A}=-\operatorname{diag}\left(0, \frac{a_{p 1,1}}{\tau_{p}^{\text {surf }}}, \cdots, \frac{a_{p 1, N_{p 1}}}{\tau_{p}^{\text {surf }}}, \frac{a_{p 2,1}}{\tau_{p}^{\text {surf }}}, \cdots, \frac{a_{p 2, N_{p 2}}}{\tau_{p}^{\text {surf }}}, \frac{a_{p 3,1}}{\tau_{p}^{\mathrm{Pt}}}, \cdots, \frac{a_{p 3, N_{p 3}}}{\tau_{p}^{\mathrm{Pt}}}, \frac{a_{p 4,1}}{\tau_{p}^{\mathrm{Pt}}}, \cdots, \frac{a_{p 4, N_{p 4}}}{\tau_{p}^{\mathrm{Pt}}}, \frac{a_{e, 1}}{\tau_{e}}, \cdots, \frac{a_{e, N_{e}}}{\tau_{e}}\right) \\
\mathbf{B}=-\frac{1}{F A}\left(\frac{1}{L_{p}}, \frac{b_{p 1,1}}{L_{p}}, \cdots, \frac{b_{p 1, N_{p 1}}}{L_{p}}, \frac{t_{p}^{+, \text {surf }}}{2} \frac{b_{p 2,1}}{L_{p}}, \cdots, \frac{t_{p}^{+, \text {surf }}}{2} \frac{b_{p 2, N_{p 2}}}{L_{p}}, \frac{b_{p 3,1}}{L_{p}}, \cdots, \frac{b_{p 3, N_{p 3}}}{L_{p}},\right. \\
\left.\frac{t_{p}^{+, \mathrm{Pt}}}{2} \frac{b_{p 4,1}}{L_{p}}, \cdots, \frac{t_{p}^{+, \mathrm{Pt}}}{2} \frac{b_{p 4, N_{p 4}}}{L_{p}}, \frac{t_{e}^{-} b_{e, 1}}{2 L_{e}}, \cdots, \frac{t_{e}^{-} b_{e, N_{e}}}{2 L_{e}}\right)^{\top}
\end{gathered}
$$

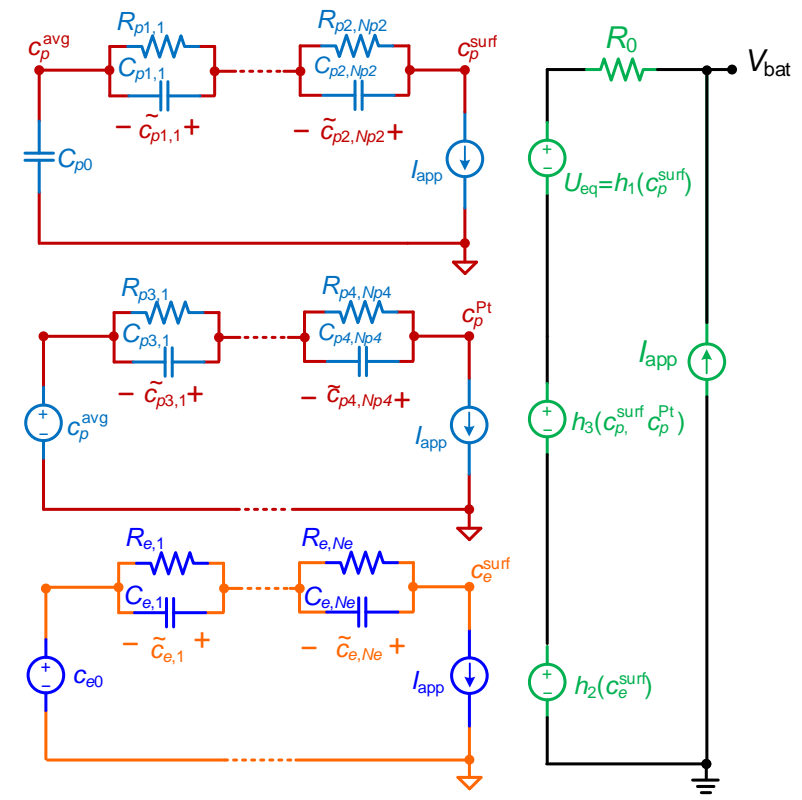

Fig. 5. Physics-based equivalent circuits for the developed ROM of ASSBs.

functions of the electrochemical parameters, cell dimension, and the PFE coefficients, i.e.,

$$
\begin{aligned}
C_{p 0} & =F A L_{p}, \\
C_{p 1, i} & =\frac{F A L_{p}}{b_{p 1, i}}, R_{p 1, i}=\frac{b_{p 1, i} \tau_{p}^{\text {surf }}}{a_{p 1, i} F A L_{p}} \quad \forall i \in \mathcal{N}_{p 1} \\
C_{p 2, i} & =\frac{2 F A L_{p}}{t_{p}^{+, \text {surf }} b_{p 2, i}}, R_{p 2, i}=\frac{t_{p}^{+, \text {surf }} b_{p 2, i} \tau_{p}^{\text {surf }}}{2 a_{p 2, i} F A L_{p}} \quad \forall i \in \mathcal{N}_{p 2} \\
C_{p 3, i} & =\frac{F A L_{p}}{b_{p 3, i}}, R_{p 3, i}=\frac{b_{p 3, i} \tau_{p}^{\mathrm{Pt}}}{a_{p 3, i} F A L_{p}} \quad \forall i \in \mathcal{N}_{p 3} \\
C_{p 4, i} & =\frac{2 F A L_{p}}{t_{p}^{+, \mathrm{Pt}} b_{p 4, i}}, R_{p 4, i}=\frac{t_{p}^{+, \mathrm{Pt}} b_{p 4, i} \tau_{p}^{\mathrm{Pt}}}{2 a_{p 4, i} F A L_{p}} \quad \forall i \in \mathcal{N}_{p 4} \\
C_{e, i} & =\frac{2 F A L_{e}}{t_{e}^{-} b_{e, i}}, R_{e, i}=\frac{t_{e}^{-} b_{e, i} \tau_{e}}{2 a_{e, i} F A L_{e}} \quad \forall i \in \mathcal{N}_{e} \\
R_{0} & =R_{e}^{\mathrm{avg}}+R_{p}^{\mathrm{avg}}+R_{p}^{\mathrm{ct}}+R_{n}^{\mathrm{ct}}
\end{aligned}
$$

where $\tau_{p}^{\text {surf }}, \tau_{p}^{\mathrm{Pt}}, \tau_{e}, t_{p}^{+ \text {,surf }}, t_{p}^{+, \mathrm{Pt}}, t_{e}^{-}, R_{e}^{\mathrm{avg}}, R_{p}^{\mathrm{avg}}, R_{p}^{\mathrm{ct}}$, and $R_{n}^{\mathrm{ct}}$ are parameters that have been defined previously.

\section{F. Observability Analysis}

One of the major use for the ROM is for state observer design. An observability analysis is necessary before any reliable design of a state observer. The general procedure to check the observability of a nonlinear battery model from the voltage measurements in the linear sense is as follows.

1) Linearize the ODEs about the states at the equilibrium to produce the system matrix $\mathbf{A}$.

2) Linearize the nonlinear output equation at the equilibrium to produce the output matrix $\mathbf{C}$.

3) Compute the observability matrix for the pair $(\mathbf{A}, \mathbf{C})$ and check the rank.

Since the state equation is already in a linear form and the system matrix is in a diagonal form, one only has to check the linearized output equation, characterized by the output matrix $\mathbf{C}$ given as

$$
\begin{aligned}
\mathbf{C}= & {[\frac{\partial h}{\partial c_{p}^{\text {avg }},}, \overbrace{\frac{\partial h}{\partial \tilde{c}_{p 1,1}}, \cdots, \frac{\partial h}{\partial \tilde{c}_{p 2, N_{p 2}}}}^{N_{p 1}+N_{p 2}},} \\
& \overbrace{\frac{\partial h}{\partial \tilde{c}_{p 3,1}}, \cdots, \frac{\partial h}{\partial \tilde{c}_{p 4, N_{p 4}}}, \overbrace{\frac{\partial h}{\partial \tilde{c}_{e, 1}}, \cdots, \frac{\partial h}{\partial \tilde{c}_{e, N_{e}}}}^{N_{p}+N_{p 4}}}^{N_{e}}]
\end{aligned}
$$

For such a linearized system in the modal canonical form, the condition when the system is fully observable from the output measurement are reduced to [28]:

1) the elements in $\mathbf{A}$ (eigenvalues) are not repeated, and

2) none of the elements in $\mathbf{C}$ are zero.

Regarding the first condition, from (33), it can be seen that the elements in the diagonal are determined by the PFE coefficients $\left(a_{p 1, i}, a_{p 2, i}, a_{p 3, i}, a_{p 4, i}\right.$, and $\left.a_{e, i}\right)$ and the time constants $\left(\tau_{p}^{\text {surf }}, \tau_{p}^{\mathrm{Pt}}\right.$, and $\left.\tau_{e}\right)$. Usually, the PFE coefficients are not the same, and only when $N_{p 2}=N_{p 4}=N_{e}$, the coefficients $a_{p 2, i}=a_{p 4, i}=a_{e, i}$ are equal for the same $i$. Even in this condition, the different time constants $\tau_{p}^{\text {surf }}, \tau_{p}^{\mathrm{Pt}}$, and $\tau_{e}$ determined by different local diffusion coefficients will in practice prevent the presence of repeated elements in $\mathbf{A}$.

For the second condition, considering $\partial U_{\text {eq }} / \partial c_{p}<0, c_{p}^{\text {surf }}>$ $0, c_{p}^{\mathrm{Pt}}>0$, and $2 c_{e 0}>c_{e}^{\text {surf }}>0$, the following relationships hold

$$
\frac{\partial h}{\partial c_{p}^{\text {avg }}}=\frac{\partial h}{\partial \tilde{c}_{p 1,1}}=\cdots=\frac{\partial h}{\partial \tilde{c}_{p 1, N_{p 1}}}=\frac{\partial h_{1}}{\partial c_{p}^{\text {surf }}}+\frac{\partial h_{3}}{\partial c_{p}^{\text {surf }}}
$$




$$
\begin{aligned}
& =\frac{\partial U_{\mathrm{eq}}}{\partial c_{p}}-\frac{2 R T t_{p}^{-}}{F c_{p}^{\text {surf }}}<0 \\
\frac{\partial h}{\partial \tilde{c}_{p 3,1}} & =\cdots=\frac{\partial h}{\partial \tilde{c}_{p 4, N_{p 4}}}=\frac{\partial h_{3}}{\partial c_{p}^{\mathrm{Pt}}}=\frac{2 R T t_{p}^{-}}{F c_{p}^{\mathrm{Pt}}}>0 \\
\frac{\partial h}{\partial \tilde{c}_{e, 1}} & =\cdots=\frac{\partial h}{\partial \tilde{c}_{e, N_{e}}}=\frac{\partial h_{2}}{\partial c_{p}^{\text {surf }}} \\
& =\frac{2 R T t_{e}^{-}}{F} \frac{2 c_{e 0}}{c_{e}^{\text {surf }}\left(2 c_{e 0}-c_{e}^{\text {surf }}\right)}>0 .
\end{aligned}
$$

With (36) and (37), it can be seen that the elements in $\mathbf{C}$ are non-zero, and thus we conclude that the ROM (32) is fully observable from the voltage measurement in the linear sense.

\section{ILlustrative Examples}

\section{A. System Configuration and Model Validation}

The PDE-based ASSB model (1)-(11) will be used as a benchmark for verification of the accuracy of the proposed ROMs. The model parameters used in this work are given in Table III. The parameters are identified with a commercial $0.7 \mathrm{mAh}$ ASSB cell based on the experimental results given in [23]. The active material of the positive electrode is lithium cobalt oxide (LCO) and the solid electrolyte is lithium phosphorus oxynitride (LiPON). Most of the cell parameters are directly obtained from [23]. Three nonlinear functions, including the equilibrium potential curve $h_{1}(\cdot)$ in (6) and two diffusion coefficient curves, i.e., $f_{D+}(\cdot)$ and $f_{D-}(\cdot)$, are fitted using the experimental results presented in [23]. These functions are given as (38)-(40), where $\theta=c_{p} / c_{p}^{\max }$ is the normalized concentration in the LCO electrode. The fitted curves are compared with the experimental data in Fig. 6. On the other hand, we notice that the parameters regarding the LiPON solid electrolyte in [23] were identified by assuming that a strong charge generation effect exist so that $r$ in (1a) is not negligible. However, in the present work, the relevant electrolyte parameters are taken from [22] where a weak charge generation effect is considered in agreement with the assumption made in Section II. In addition, the diffusion coefficient $D_{e}^{+}$is the only parameter tuned manually to fit the experimental data. All models including the benchmark model and different ROMs were implemented in MATLAB $\mathrm{R} 2016 \mathrm{~b}$, discretized in the time domain with the backward Euler method and sampled with $1 \mathrm{~s}$, and the simulated results were obtained on a 64-bit Windows 10 on a PC, with Intel Corei7-4790 CPU@ 3.60 Hz and 16GB RAM.

The benchmark PDE model was spatially discretized using the FVM [29] and both the electrolyte and the electrode domains were evenly divided into 100 and 30 control volumes, respectively.The simulated discharge curves of the terminal voltage under various constant current rates are plotted in Fig. 7 against the experimental data extracted from [23], for comparison. It is shown that the model can accurately capture the dynamics up to until $6 \mathrm{C}$ with increasing model errors with increasing current rate. The model accuracy can be further improved by global optimization of more tunable parameters, but we leave that relevant investigation to our future work.

In the next two subsections, the system orders of the proposed ROMs are first selected empirically as $N_{e}=5$ and
TABLE III

ASSB MODEL PARAMETERS

\begin{tabular}{ccc}
\hline \hline Symbol & Unit & Value \\
\hline$F$ & {$\left[\mathrm{C} \cdot \mathrm{mol}^{-1}\right]$} & 96,485 \\
$R$ & {$\left[\mathrm{~J} \cdot \mathrm{K}^{-1} \cdot \mathrm{mol}^{-1}\right]$} & 8.314 \\
$T$ & {$[\mathrm{~K}]$} & $2933^{\mathrm{a}}$ \\
$A$ & {$\left[\mathrm{~m}^{2}\right]$} & $3.36 \times 10^{-4}$ \\
$Q_{\max 0}$ & {$[\mathrm{mAh}]$} & $0.7 \times 10^{-4}$ \\
$L_{e}$ & {$[\mathrm{~m}]$} & $3.62 \times 10^{-6}$ \\
$L_{p}$ & {$[\mathrm{~m}]$} & $8.08 \times 10^{-6}$ \\
$D_{e}^{+}$ & {$\left[\mathrm{m}^{2} \cdot \mathrm{s}^{-1}\right]$} & $1.8 \times 10^{-14} \mathrm{a}$ \\
$D_{e}^{-}$ & {$\left[\mathrm{m}^{2} \cdot \mathrm{s}^{-1}\right]$} & $2.2 \times 10^{-16} \mathrm{~b}$ \\
$c_{0}$ & {$\left[\mathrm{~mol}^{-1} \mathrm{~m}^{-3}\right]$} & $62,000^{\mathrm{b}}$ \\
$\delta$ & $\left.-\mathrm{m}^{-3}\right]$ & $0.04^{\mathrm{b}}$ \\
$c_{p}^{\max }$ & {$\left[\mathrm{mol} \cdot \mathrm{m}^{-3}\right]$} & 32,200 \\
$c_{n}^{\text {avg }}$ & {$\left[\mathrm{mol} \cdot \mathrm{m}^{-3}\right]$} & 76,400 \\
$c_{p}^{0 \%}$ & {$\left[\mathrm{~mol} \cdot \mathrm{m}^{-3}\right]$} & 26,404 \\
$c_{p}^{100 \%}$ & {$\left[\mathrm{~mol} \cdot \mathrm{m}^{-3}\right]$} & 16,744 \\
$k_{0, p}$ & {$\left[\mathrm{~m}^{2.5} \cdot \mathrm{mol}^{-0.5} \cdot \mathrm{s}^{-1}\right]$} & $1.53 \times 10^{-11}$ \\
$k_{0, n}$ & {$\left[\mathrm{~m} \cdot \mathrm{s}^{-1}\right]$} & $1.09 \times 10^{-9}$ \\
\hline \hline
\end{tabular}

a Adjusted from [22]

b Obtained from [22]

Other parameters are obtained from [23]
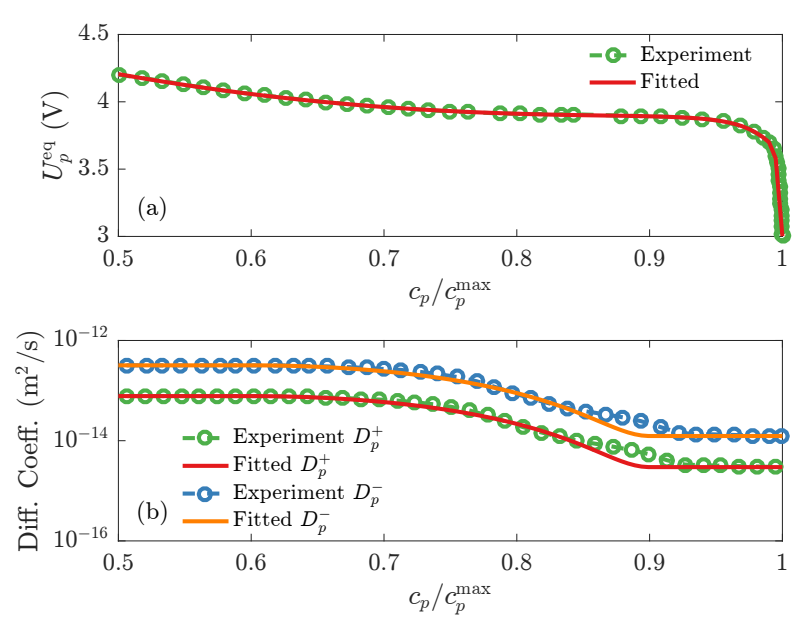

Fig. 6. Experimental and fitted curves of (a) equilibrium potential and (b) diffusion coefficients of the positive electrode.

$N_{p 1}=N_{p 2}=N_{p 3}=N_{p 4}=3$ based on the observation of Fig. 2 to Fig. 4. Since the sampling rate and discrete time step is $1 \mathrm{~s}$, it is not motivated to approximate much higher frequency components than $1 \mathrm{~Hz}$ by increasing the model orders and detailed investigation on the effects of model order will be provided in Section IV-D.

\section{B. Comparison Under Constant Discharge Current}

Fig. 8 shows a comparison of the results under a 1C constant current discharge with different models, including the benchmark PDE (FVM) model, the proposed PFE-MM model with and without the correction, as well as a ROM proposed in [20], where the diffusion equations are reduced with Padé approximation and the mass-transfer overpotential is solved with a polynomial approximation. The simulated cells were initially fully charged at $4.2 \mathrm{~V}$ and discharge was stopped at $3.0 \mathrm{~V}$. As seen in Fig. 8(a), at $t=0$, there is an 


$$
\begin{gathered}
U_{p}^{\mathrm{eq}}=h_{1}(\theta)=\frac{245.67-909.73 \theta+1370.02 \theta^{2}-1109.36 \theta^{3}+519.2 \theta^{4}-115.81 \theta^{5}}{42.317-122.12 \theta+112.28 \theta^{2}-27.473 \theta^{3}-5.02 \theta^{4}} \\
D_{p}^{+}=f_{D+}(\theta)= \begin{cases}7.76 \times 10^{-14}, & \theta<0.6 \\
3.7315 \times 10^{-14} \cos (10.472 \theta)+4.0285 \times 10^{-14}, & 0.6 \leq \theta \leq 0.9 \\
2.97 \times 10^{-15}, & \theta>0.9\end{cases} \\
D_{p}^{-}=f_{D_{-}}(\theta)= \begin{cases}3.19 \times 10^{-13}, & \theta<0.6 \\
1.4330 \times 10^{-13} \cos (10.472 \theta)+1.6570 \times 10^{-13}, & 0.6 \leq \theta \leq 0.9 \\
1.24 \times 10^{-14}, & \theta>0.9\end{cases}
\end{gathered}
$$

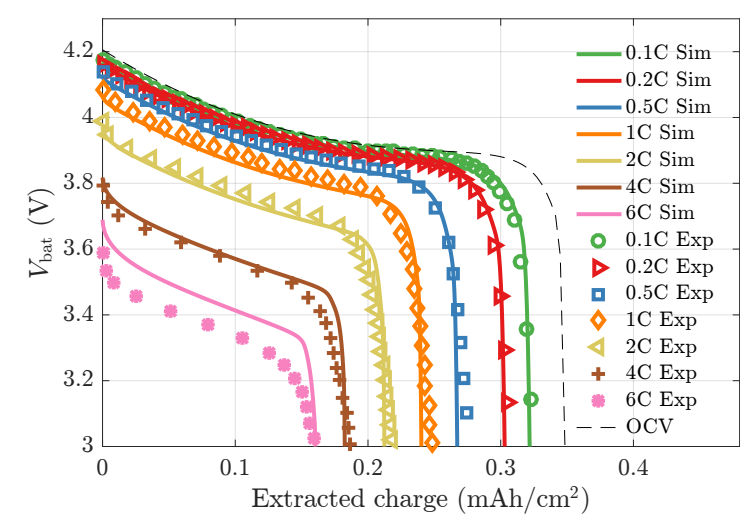

Fig. 7. Comparison of simulated terminal voltages (Sim) and experimental data (Exp) for an ASSB cell under various discharge current rates.
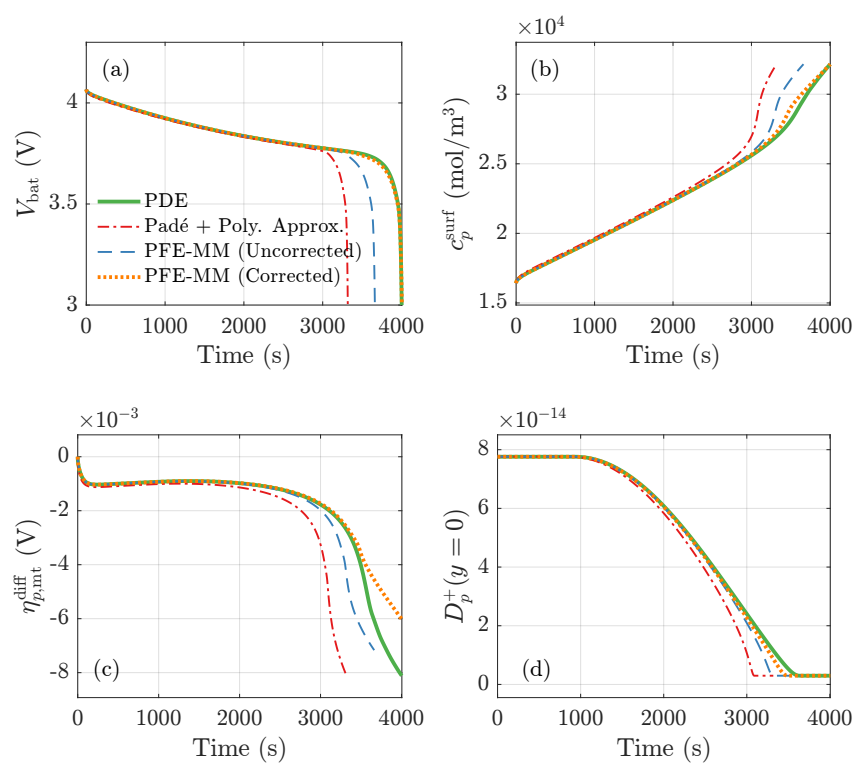

Fig. 8. Simulated results under $1 \mathrm{C}$ constant current discharge profile. (a) Voltage. (b) Surface concentration at $y=0$. (c) Mass-transfer diffusion overpotential in the positive electrode. (d) Diffusion coefficient.

immediate voltage drop of $0.07 \mathrm{~V}$ due to the presence of the series-connected resistance $R_{0}$, as shown in Fig. 5 . It can be observed in Fig. 8 that by setting the correction coefficient $k=0.31$ in (27), the proposed PFE-MM model compares very favorably to that of the PDE model implementations. As seen in Fig. 8(d), the reduction in the diffusion coefficient is accurately captured, and this results in accurate prediction of the voltage, concentration, as well as the overpotential in Fig. 8(a) to Fig. 8(c). On the contrary, when there is no correction, there is an accumulated error on the diffusion coefficient and the drop at the end of discharge, which lead to considerable prediction error for the knee point, as seen in Fig. 8(a): The end of discharge is $338 \mathrm{~s}$ earlier than the benchmark. Furthermore, the ROM in [20] exhibits even larger errors in predicting the knee point (683 $\mathrm{s}$ earlier) due to its ignorance of the effect of ionic migration in the positive electrode, i.e., the mass-transfer overpotential $\eta_{p}^{\mathrm{mt}}$ of the positive electrode in (29) is considered zero in [20]. Note that the discharge time under $1 \mathrm{C}$ current is longer than $1 \mathrm{~h}$, since the $0 \%$ SOC is defined at $c_{p}^{0 \%} / c_{p}^{\max }=0.82$ rather than $\theta=1$, and the corresponding equilibrium voltage is about $3.86 \mathrm{~V}$ [23].

TABLE IV

CPU Time AND Accuracy of ASSB Cell Voltage With Different MODELS

\begin{tabular}{lccccc}
\hline \hline \multirow{2}{*}{ Model } & $1 \mathrm{C}$ & $4 \mathrm{C}$ & \multicolumn{3}{c}{ Modified FUDS } \\
\cline { 2 - 6 } & Time & Time & Time & RMSE & MAX \\
\hline PDE (FVM) & $17.5 \mathrm{~s}$ & $4.3 \mathrm{~s}$ & $36.3 \mathrm{~s}$ & - & - \\
Padé + Poly. & $0.9 \mathrm{~s}$ & $0.2 \mathrm{~s}$ & $2.1 \mathrm{~s}$ & $4.9 \mathrm{mV}$ & $2.26 \mathrm{~V}$ \\
PFE-MM (Uncorr.) & $1.1 \mathrm{~s}$ & $0.3 \mathrm{~s}$ & $2.7 \mathrm{~s}$ & $2.2 \mathrm{mV}$ & $0.61 \mathrm{~V}$ \\
PFE-MM (Corr.) & $1.1 \mathrm{~s}$ & $0.3 \mathrm{~s}$ & $2.8 \mathrm{~s}$ & $0.7 \mathrm{mV}$ & $0.05 \mathrm{~V}$ \\
\hline \hline
\end{tabular}

Fig. 9 shows a similar comparison to Fig. 8, except that a 4C discharge current was applied. Indeed, at a higher current rate, the simulated error increases: The root-meansquare error (RMSE) of the voltage increases from $1.9 \mathrm{mV}$ for $1 \mathrm{C}$ discharge to $7.6 \mathrm{mV}$, but the accuracy of the proposed model still compares favorably to the PDE model, and terminal voltages, surface concentration, and diffusion overpotentials are all adequately predicted.

The recorded CPU times for the constant current discharge examples are summarized in Table IV. It can be seen that the proposed ROMs are much more computationally efficient than the benchmark PDE model solved using the FVM. Compared to the ROM developed in [20], the accuracy of the proposed ROMs were significantly improved at the expense of slightly increased computation time to solve the enhanced electrode diffusion and migration equations, while the need for numerical integration is avoided to solve the mass-transfer overpotentials.

\section{Comparison Under Dynamic Driving Cycling Test}

Next, in order to test the model performance under dynamic operating conditions, a modified Federal Urban Driving Sched- 

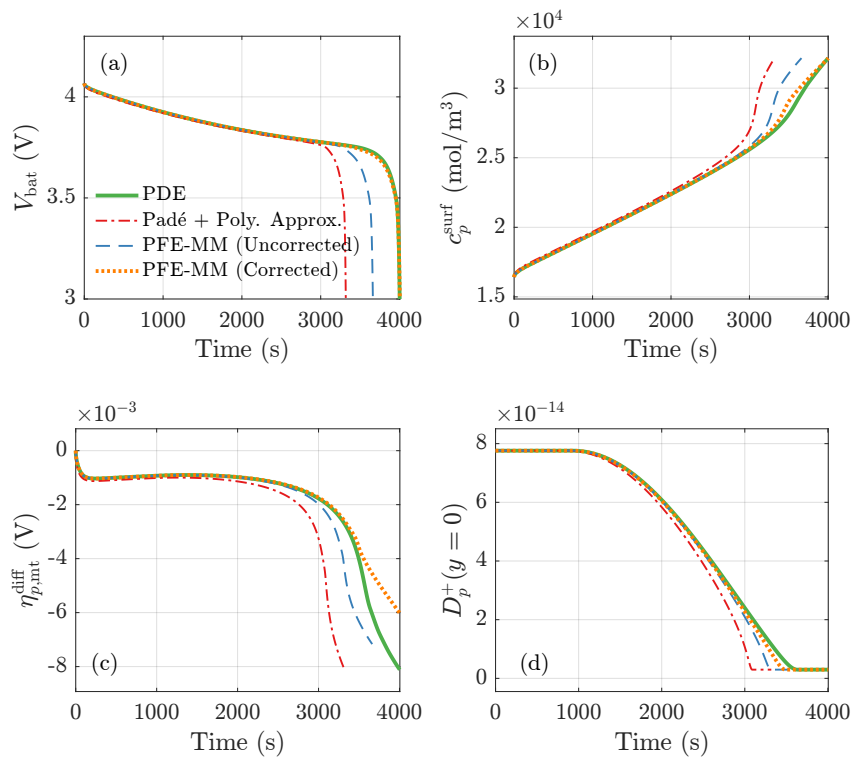

Fig. 9. Simulated results under $4 \mathrm{C}$ constant current discharge profile. (a) Voltage. (b) Surface concentration at $y=0$. (c) Mass-transfer diffusion overpotential in the positive electrode. (d) Diffusion coefficient.
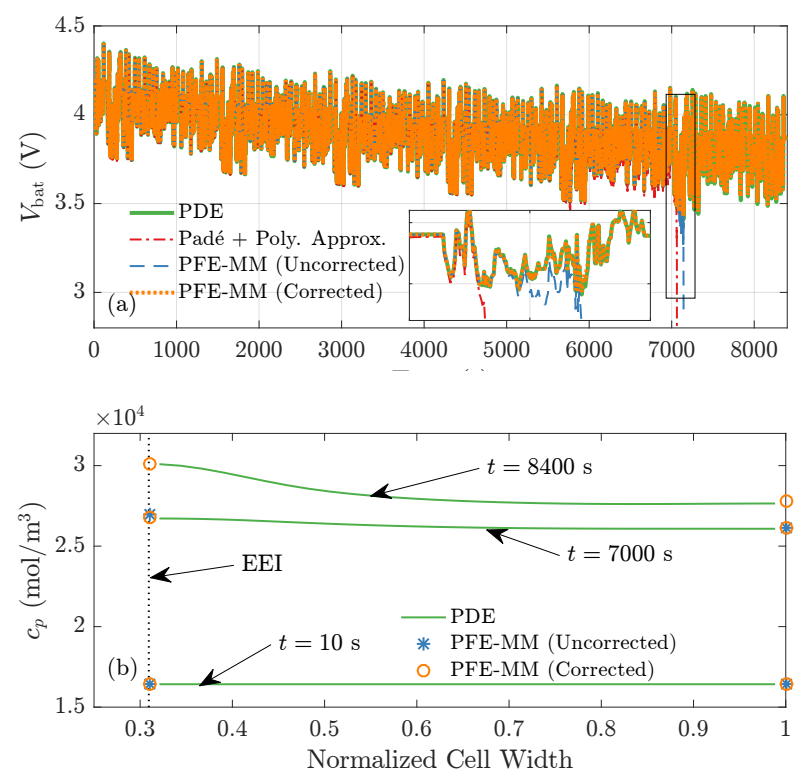

Fig. 10. Simulated results under a modified FUDS current profile. (a) Cell voltage. (b) Distributions of the concentration in the positive electrode.

ule (FUDS) test profile was chosen as the input current. The original maximum current rate is $1 \mathrm{C}$ and the magnitude of the profile is amplified by a factor of 4 . The simulated ASSB voltage and surface concentration are shown in Fig. 10. Again, the proposed ROM can accurately reproduce the PDE results after introducing the correction factor and from Table IV, it can be seen the RMSE of the predicted voltage using the corrected $\mathrm{ROM}$ is lower than $1 \mathrm{mV}$, while the maximum error (MAX) is about $50 \mathrm{mV}$. In contrast, from Fig. 10(a), it can be seen that the simulations of both the ROM based on [20] and the uncorrected PFE model terminated at about $t=7130 \mathrm{~s}$ since the voltage erroneously hits the preset cutoff voltage of $3.0 \mathrm{~V}$,
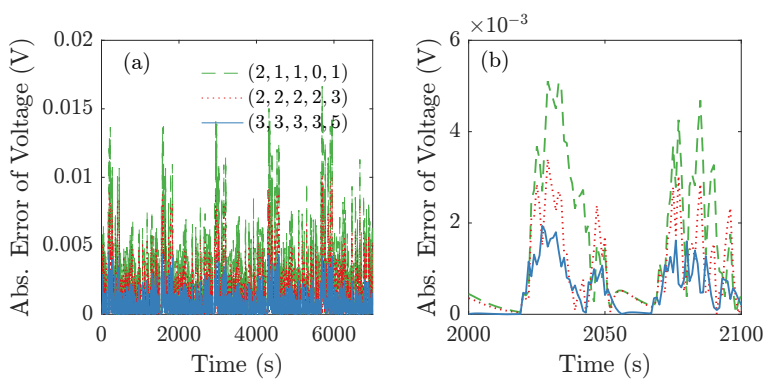

Fig. 11. Model errors under a modified FUDS profile with different model orders. (a) Voltage error. (b) Voltage error between 2000 and 2100 s. In the legend, the numbers in the bracket indicate $\left(N_{p 1}, N_{p 2}, N_{p 3}, N_{p 4}, N_{e}\right)$.

leading to high MAX. In addition, Fig. 10(b) shows that the proposed method is superior in the avoidance of approximating the entire concentration distribution in the positive electrode, while the boundary values are accurately predicted.

\section{Effect of Model Order}

Fig. 11 compares the model accuracy using different model orders under a similar modified FUDS current profile. In general, increasing the system order of the PFE model can improve the high-frequency response, as indicated in the Bode diagrams in Fig. 2 to Fig. 4. However, there is no need to adopt very high-order approximations, since usually the energy contained in the high-frequency components of the input current is not very high for most battery management applications. For example, most dynamic current test profiles are given with a sampling time interval of $1 \mathrm{~s}$, which means that $1 \mathrm{~Hz}$ can be considered the highest frequency to be complied with by a reduced model. As can be seen from Fig. 11, under the same enhanced FUDS profile with the maximum current rate of $4 \mathrm{C}$, compared to the high-order PFE model $\left(N_{p 1}=N_{p 2}=N_{p 3}=N_{p 4}=3\right.$ and $\left.N_{e}=5\right)$ with an RMSE of $0.7 \mathrm{mV}$ (as given in Table IV), a much low-order PFE model with $N_{p 1}=2, N_{p 2}=1, N_{p 3}=1, N_{p 4}=0, N_{e}=1$ is less accurate but can still regenerate the battery behaviors sufficiently well with an RMSE of $2.5 \mathrm{mV}$. Note that $N_{p 3}$ and $N_{p 4}$ are used to model the effect of the concentrations on the current collector side of the positive electrode, and in many cases they are ignorable for light load applications. Hence, it is seen that a 5th-order model might be sufficiently accurate for battery management.

\section{CONCLUSIONS}

An order reduction method is proposed to simplify the PDE-based all solid-phase battery model into an ODE model. The specific contributions include: 1) a generic reducedorder model based on partial fraction expansion and moment matching is proposed for control system design, and it is also formulated into an equivalent circuit form; 2) Important nonlinear phenomena under high rate operation, including the concentration-dependent diffusion and the ionic migration processes in the positive electrode, are properly addressed. The simplified model is linear time-varying in terms of its state equation but nonlinear in its output equation, and we show 
that in practice the reduced-order model is fully observable in the linear sense. The model can be readily implemented for online management functionalities to achieve model-based state estimation, parameter identification, and optimal control.

\section{APPENDIX}

\section{A. Derivation of Electrolyte Diffusion Transfer Function}

The general form of the Laplace transform of (1a) is

$$
c_{e}(x, s)=A \sinh (\sqrt{s} x / \alpha)+B \cosh (\sqrt{s} x / \alpha)
$$

where $A$ and $B$ are two coefficients, $\alpha=\sqrt{D_{e}^{\text {eff }}}$, and the term $r$ in (1a) is ignored. The gradient of (41) is

$$
\frac{\partial c_{e}(x, s)}{\partial x}=\frac{A \sqrt{s}}{\alpha} \cosh \left(\frac{\sqrt{s} x}{\alpha}\right)+\frac{B \sqrt{s}}{\alpha} \sinh \left(\frac{\sqrt{s} x}{\alpha}\right)
$$

Using (42) and the boundary conditions (12), one can solve for the coefficients $A$ and $B$, both of which are proportional to the current density $i_{\text {app }}$, i.e,

$$
\frac{A}{i_{\text {app }}(s)}=\frac{\alpha t_{e}^{-}}{D_{e}^{\text {eff }} \sqrt{s}}, \quad \frac{B}{i_{\text {app }}(s)}=-\frac{\alpha t_{e}^{-}}{D_{e}^{\text {eff }} \sqrt{s}} \tanh \left(\frac{\sqrt{s} L_{e}}{2 \alpha}\right)
$$

Substituting (43) into (41) and reorganizing the equation, we obtain the transcendental transfer function (13) for the electrolyte diffusion equation.

\section{B. Derivation of the Simplified Mass-Transfer Overpotential}

Considering two identities

$$
\begin{gathered}
\frac{\partial c_{e}(x, t)}{\partial x}=c_{e}(x, t) \frac{\partial \ln c_{e}(x, t)}{\partial x} \\
\int_{0}^{L_{e}}\left(\frac{\partial \ln c_{e}(x, t)}{\partial x}\right) d x=\ln \left(\frac{c_{e}\left(L_{e}, t\right)}{c_{e}(0, t)}\right)
\end{gathered}
$$

First, substituting (44) into (3), one yields

$$
E(x, t)=-\frac{i_{\mathrm{app}}(t)}{\kappa_{e}^{\mathrm{eff}}(x, t)}+\frac{R T}{F}\left(\frac{D_{e}^{+}-D_{e}^{-}}{D_{e}^{+}+D_{e}^{-}}\right) \frac{\partial \ln c_{e}(x, t)}{\partial x}
$$

where $\kappa_{e}^{\text {eff }}:=\left(D_{e}^{+}+D_{e}^{-}\right)\left(\frac{F^{2}}{R T}\right) c_{e}$ represents the local conductivity in the solid electrolyte.

Substituting (46) into (2) yields

$$
\begin{aligned}
\eta_{e}^{\mathrm{mt}}(t)= & \frac{R T}{F} \ln \left(\frac{c_{e}\left(L_{e}, t\right)}{c_{e}(0, t)}\right)+\int_{0}^{L_{e}}\left(\frac{i_{\mathrm{app}}(t)}{\kappa_{e}^{\mathrm{eff}}(x, t)}\right) d x+ \\
& \frac{R T}{F}\left(\frac{D_{e}^{+}-D_{e}^{-}}{D_{e}^{+}+D_{e}^{-}}\right) \int_{0}^{L_{e}}\left(\frac{\partial \ln c_{e}(x, t)}{\partial x}\right) d x
\end{aligned}
$$

We define the volume-averaged electrolyte resistance as

$$
R_{e}^{\mathrm{avg}}:=\frac{1}{A} \int_{0}^{L_{e}}\left(\frac{1}{\kappa_{e}^{\mathrm{eff}}(x, t)}\right) d x \approx \frac{L_{e}}{A \bar{\kappa}_{e}^{\mathrm{eff}}}
$$

where $\bar{\kappa}_{e}^{\text {eff }}:=\left(D_{e}^{+}+D_{e}^{-}\right)\left(\frac{F^{2} A}{R T}\right) c_{e 0}$ is the average electrolyte conductivity and $A$ is the cross-sectional area of the electrode. Substituting (48) and (45) into (47), thus

$$
\eta_{e}^{\mathrm{mt}}(t) \approx \frac{2 R T t_{e}^{-}}{F} \ln \left(\frac{c_{e}\left(L_{e}, t\right)}{c_{e}(0, t)}\right)+R_{e}^{\mathrm{avg}} I_{\mathrm{app}}(t)
$$

where $t_{e}^{-}=D_{e}^{-} /\left(D_{e}^{+}+D_{e}^{-}\right)$and $I_{\text {app }}=A i_{\text {app }}$.

Finally, with $c_{e}\left(L_{e}, t\right)=c_{e}^{\text {surf }}(t)$ and $c_{e}(0, t)=2 c_{e 0}-$ $c_{e}^{\text {surf }}(t)$, the expression of the mass-transfer overpotential (28) is obtained. Based on a similar procedure, the simplified expression of the mass-transfer overpotential (29) can be derived from (7) and (8) for the positive electrode.

\section{REFERENCES}

[1] M. T. Lawder, B. Suthar, P. W. C. Northrop, S. De, C. M. Hoff, O. Leitermann, M. L. Crow, S. Santhanagopalan, and V. R. Subramanian, "Battery energy storage system (BESS) and battery management system (BMS) for grid-scale applications," Proc. IEEE, vol. 102, no. 6, pp. 1014-1030, Jun. 2014.

[2] Y. Li, M. Vilathgamuwa, S. S. Choi, B. Xiong, J. Tang, Y. Su, and Y. Wang, "Design of minimum cost degradation-conscious lithium-ion battery energy storage system to achieve renewable power dispatchability," Appl. Energy, vol. 260, p. 114282, Feb. 2020.

[3] Y. Li, B. Xiong, D. M. Vilathgamuwa, Z. Wei, C. Xie, and C. Zou, "Constrained ensemble Kalman filter for distributed electrochemical state estimation of lithium-ion batteries," IEEE Trans. Ind. Informat., vol. 17, no. 1, pp. 240-250, Jan. 2021.

[4] Q. Wang, P. Ping, X. Zhao, G. Chu, J. Sun, and C. Chen, "Thermal runaway caused fire and explosion of lithium ion battery," J. Power Sources, vol. 208, pp. $210-224,2012$.

[5] P. Albertus, S. Babinec, S. Litzelman, and A. Newman, "Status and challenges in enabling the lithium metal electrode for high-energy and low-cost rechargeable batteries," Nature Energy, vol. 3, no. 1, pp. 16-21, Jan. 2018.

[6] A. C. Luntz, J. Voss, and K. Reuter, "Interfacial challenges in solid-state Li ion batteries," J. Phys. Chem. Lett., vol. 6, no. 22, pp. 4599-4604, Nov. 2015.

[7] S. Randau, D. A. Weber, O. Kötz, R. Koerver, P. Braun, A. Weber, E. Ivers-Tiffée, T. Adermann, J. Kulisch, W. G. Zeier, F. H. Richter, and J. Janek, "Benchmarking the performance of all-solid-state lithium batteries," Nature Energy, vol. 5, no. 3, pp. 259-270, Mar. 2020.

[8] M. Bahramipanah, D. Torregrossa, R. Cherkaoui, and M. Paolone, "Enhanced equivalent electrical circuit model of lithium-based batteries accounting for charge redistribution, state-of-health, and temperature effects," IEEE Trans. Transport. Electrific., vol. 3, no. 3, pp. 589-599, 2017.

[9] C. Zou, C. Manzie, and D. Nesic, "A framework for simplification of PDE-based lithium-ion battery models," IEEE Trans. Control Syst. Technol., vol. 24, no. 5, pp. 1594-1609, Sep. 2016.

[10] R. Gu, P. Malysz, H. Yang, and A. Emadi, "On the suitability of electrochemical-based modeling for lithium-ion batteries," IEEE Trans. Transport. Electrific., vol. 2, no. 4, pp. 417-431, Dec. 2016.

[11] P. Ramadass, B. Haran, P. M. Gomadam, R. White, and B. N. Popov, "Development of first principles capacity fade model for Li-ion cells," J. Electrochem. Soc., vol. 151, no. 2, pp. A196-A203, Jan. 2004.

[12] X. Jin, A. Vora, V. Hoshing, T. Saha, G. Shaver, R. E. Garca, O. Wasynczuk, and S. Varigonda, "Physically-based reduced-order capacity loss model for graphite anodes in Li-ion battery cells," J. Power Sources, vol. 342, pp. 750-761, Feb. 2017.

[13] X.-G. Yang, Y. Leng, G. Zhang, S. Ge, and C.-Y. Wang, "Modeling of lithium plating induced aging of lithium-ion batteries: Transition from linear to nonlinear aging," J. Power Sources, vol. 360, pp. 28-40, Aug. 2017.

[14] Y. Li, D. Karunathilake, D. M. Vilathgamuwa, Y. Mishra, T. W. Farrell, S. S. Choi, and C. Zou, "Model order reduction techniques for physicsbased lithium-ion battery management: A survey," IEEE Ind. Electron. Mag., in press.

[15] J. Kasemchainan, S. Zekoll, D. Spencer Jolly, Z. Ning, G. O. Hartley, J. Marrow, and P. G. Bruce, "Critical stripping current leads to dendrite formation on plating in lithium anode solid electrolyte cells," Nature Mat., vol. 18, no. 10, pp. 1105-1111, Oct. 2019.

[16] R. Behrou and K. Maute, "Numerical modeling of damage evolution phenomenon in solid-state lithium-ion batteries," J. Electrochem. Soc., vol. 164, no. 12, pp. A2573-A2589, 2017.

[17] K. Becker-Steinberger, S. Funken, M. Landstorfer, and K. Urban, "A mathematical model for all solid-state lithium-ion batteries," ECS Trans., vol. 25, no. 36, pp. 285-296, Apr. 2010.

[18] D. Danilov, R. A. Niessen, and P. H. Notten, "Modeling all-solid-state Li-ion batteries," J. Electrochem. Soc., vol. 158, no. 3, pp. A215-A222, Mar. 2011. 
[19] Y. Kim, X. Lin, A. Abbasalinejad, S. U. Kim, and S. H. Chung, "On state estimation of all solid-state batteries," Electrochim. Acta, vol. 317 pp. 663-672, Sep. 2019.

20] Z. Deng, X. Hu, X. Lin, L. Xu, J. Li, and W. Guo, "A reducedorder electrochemical model for all-solid-state batteries," IEEE Trans. Transport. Electrific., vol. 7, pp. 464-473, Jun. 2021.

[21] Z. Deng, X. Hu, X. Lin, Y. Kim, and J. Li, "Sensitivity analysis and joint estimation of parameters and states for all-solid-state batteries," IEEE Trans. Transport. Electrific., vol. 7, no. 3, pp. 1314-1323, Sep. 2021.

[22] N. Kazemi, D. L. Danilov, L. Haverkate, N. J. Dudney, S. Unnikrishnan, and P. H. L. Notten, "Modeling of all-solid-state thin-film Li-ion batteries: Accuracy improvement," Solid State Ionics, vol. 334, pp. 111116, Jul. 2019.

[23] L. H. J. Raijmakers, D. L. Danilov, R. A. Eichel, and P. H. L. Notten, "An advanced all-solid-state Li-ion battery model," Electrochim. Acta, vol. 330, p. 135147, Jan. 2020.

[24] C. Sun, J. Liu, Y. Gong, D. P. Wilkinson, and J. Zhang, "Recent advances in all-solid-state rechargeable lithium batteries," Nano Energy, vol. 33, pp. 363-386, Mar. 2017.

[25] D. Danilov and P. H. L. Notten, "Mathematical modelling of ionic transport in the electrolyte of Li-ion batteries," Electrochim. Acta, vol. 53, no. 17, pp. 5569-5578, Jul. 2008

[26] T. Wik and C. Breitholtz, "Rational transfer function models for biofilm reactors," AIChE J., vol. 44, no. 12, pp. 2647-2657, 1998.

[27] A. C. Antoulas, "Approximation of large-scale dynamical systems: An overview," IFAC Proc. Vol., vol. 37, no. 11, pp. 19-28, Jul. 2004

[28] Z. Gajic and M. Leli, "Controllability and observability," in Modern Control Systems Engineering. London: Prentice Hall, 1996.

[29] Y. Li, M. Vilathgamuwa, T. Farrell, S. S. Choi, N. T. Tran, and J. Teague, "A physics-based distributed-parameter equivalent circuit model for lithium-ion batteries," Electrochim. Acta, vol. 299, pp. 451-469, Mar. 2019.

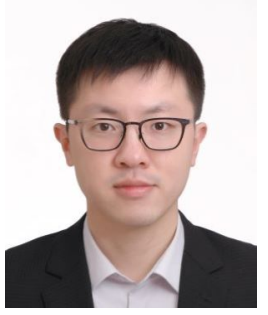

Yang Li (Member, IEEE) received the B.E. degree in electrical engineering from Wuhan University, Wuhan, China, in 2007, and the M.Sc. and Ph.D. degrees in power engineering from Nanyang Technological University (NTU), Singapore, in 2008 and 2015, respectively. From 2015 to 2018, he was a Research Fellow with the Energy Research Institute, NTU and the School of Electrical Engineering and Computer Science, Queensland University of Technology, Brisbane, QLD, Australia. Since 2019, he has been with the School of Automation, Wuhan University of Technology, Wuhan. He is currently a Researcher with the Department of Electrical Engineering, Chalmers University of Technology, Gothenburg, Sweden. His research interests include modeling and control of energy storage systems in power systems and transport sector.

Dr. Li was a recipient of the EU Marie Skłodowska-Curie Action Individual Fellowship in 2020 .

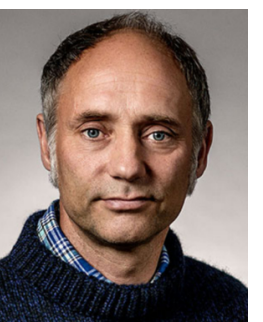

Torsten Wik received the M.Sc. degree in chemical engineering (major in applied mathematics), the Licentiate of Engineering degree in control engineering, the Ph.D. degree in environmental sciences (major in automatic control), and the Docent degree in electrical engineering from the Chalmers University of Technology, Gothenburg, Sweden, in 1994, 1996, 1999, and 2004, respectively. From 2005 to 2007, he was a Senior Researcher with Volvo Technology, Gothenburg, researching on control system design for combustion engine test cells, and combined reformer and fuel cells. He is a Professor and the Head of the Automatic Control Group, Department of Electrical Engineering, Chalmers University of Technology. His current research interests include optimal control, model reduction, and systems with model uncertainties, with applications to energy storage, environmental, and biological systems.

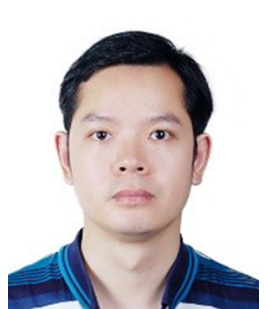

Changjun Xie (Member, IEEE) received the Ph.D. degree in vehicle engineering from the Wuhan University of Technology (WUT), Wuhan, Hubei, China, in 2009. From 2012 to 2013, he was a Visiting Scholar with the University of California, Davis, California, USA. He is currently a Professor with the School of Automation, WUT, Wuhan, China. $\mathrm{He}$ is currently the Vice Dean of the School of Automation and the Director of the Institute of New Energy Detection and Control Technology, WUT. His research interests include battery management, control, and optimization of new energy vehicles.

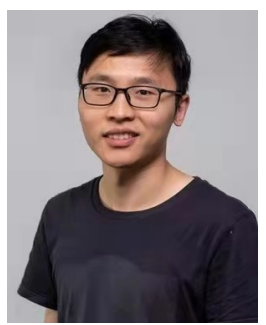

Yicun Huang received the M.Eng. degree from the University of Bristol, UK, in 2015, and the Ph.D degree from the School of Engineering, University of Canterbury, Christchurch, New Zealand, in 2020, both in mechanical engineering. He is currently a Postdoctoral researcher with the Department of Electrical Engineering, Chalmers University of Technology, Gothenburg, Sweden. His research interests include advanced modeling for energy materials.

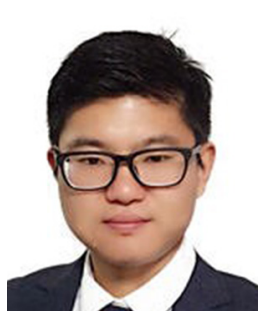

Binyu Xiong (Member, IEEE) received the B.E. degree in electrical engineering from Wuhan University, Wuhan, China, in 2010, the M.Sc. and Ph.D. degrees from the Nanyang Technological University, Singapore, in 2011 and 2016, respectively. He is currently an Associate Professor with the School of Automation, Wuhan University of Technology, Wuhan, China. His research interests include electrical and thermal modeling of batteries, battery state of charge estimation, large-scale energy storage systems, and renewable energy generations.

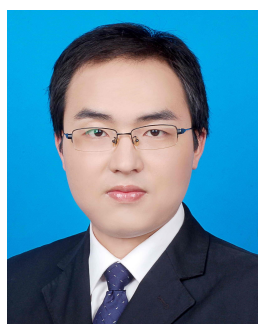

Jinrui Tang (Member, IEEE) received the B.S. degree in electrical engineering from Zhejiang University, Hangzhou, China, in 2009, and the Ph.D. degree in electrical engineering from the School of Electrical and Electronics Engineering, Huazhong University of Science and Technology, Wuhan, China, in 2014. He is currently an Associate Professor with the School of Automation, Wuhan University of Technology, Wuhan. His research interests include power system control and protection.

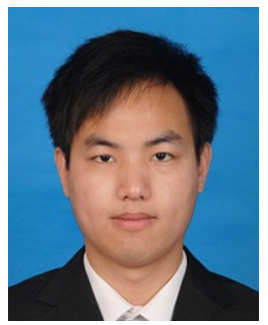

Changfu Zou (Member, IEEE) received the Ph.D. degree in automation and control engineering from the Department of Mechanical Engineering, University of Melbourne, VIC, Australia, in 2017. He was a Postdoctoral Researcher with the Chalmers University of Technology, Gothenburg, Sweden, where he is currently an Assistant Professor with the Automatic Control Group, Department of Electrical Engineering.

His current research interests include modeling and control of energy storage systems for automotive and power grid applications. 